\title{
Uma abordagem bayesiana para o método de controle "on-line" de Taguchi para atributos
}

\author{
Leyla Costa Ramos
}

DISSERTAÇÃO APRESENTADA

AO

INSTITUTO DE MATEMÁTICA E ESTATÍSTICA

DA

UNIVERSIDADE DE SÃO PAULO

PARA

OBTENÇÃO DO TÍTULO

$\mathrm{DE}$

MESTRE EM CIÊNCIAS

Área de Concentração: Estatística

Orientador: Prof. Dr. Luís Gustavo Esteves

São Paulo, maio de 2008. 


\section{Uma abordagem bayesiana para o método de controle "on-line" de Taguchi para atributos}

Este exemplar corresponde à redação final da dissertação devidamente corrigida e defendida por Leyla Costa Ramos e aprovada pela Comissão Julgadora.

Banca Examinadora:

- Prof. Dr. Luís Gustavo Esteves (orientador) - IME-USP.

- Prof. Dr. Wagner de Souza Borges - IME-USP.

- Prof. Dr. Josemar Rodrigues - UFSCAR. 
"Nós não somos o que sabemos. Somos o que estamos dispostos a aprender."

(Council on Ideas) 
À memória de meu pai João Carlos, com sua ausência pensei em desistir, mas sua lembrança me deu forças para continuar. 


\section{Agradecimentos}

Primeiramente agradeço aos meus pais. À minha mãe, Lidia, por tudo o que me ensinou e pelo apoiou incondicional em todas as minhas decisões. Ao meu pai, João Carlos, que apesar de não poder ter me acompanhado até o final desse trabalho, deixou a força de sua torcida e de seu orgulho por todas as minhas conquistas.

À minha irmã, Sheila, que sempre foi um exemplo e uma grande conselheira junto com meu cunhado, Fábio. E aos meus sobrinhos, Eduardo e Carolina que nasceram para iluminar as nossas vidas.

À meu marido, Marcelo, por estar sempre ao meu lado, me ajudando e incentivando. E aos seus pais, Lourdes e Nelson, e seus irmãos, Vanessa e Márcio, que são minha segunda família.

Aos meus amigos da Nielsen, pelo apoio desde o princípio. Em especial, ao Eduardo Alves, que sempre cobriu as minhas ausências, permitindo que eu dedicasse com tranqüilidade as horas necessárias ao Mestrado.

Aos meus amigos do IME, por todos os momentos que passamos juntos nessa jornada, em especial, aos amigos que me acompanharam na Graduação e no Mestrado, Juliana e Augusto e principalmente, para aquele que me acompanhou em todos os cantos (Graduação, Mestrado e trabalho), Luis Henrique.

E finalmente, ao meu dedicado, paciente e didático professor orientador, Luís Gustavo Esteves, sem o qual esse trabalho não teria sido realizado. 


\section{Resumo}

Nesse trabalho, apresentaremos o método econômico desenvolvido por Taguchi para monitoramento on line da qualidade para atributos. O propósito deste método é obter o intervalo de inspeção que minimiza o custo esperado por item produzido em um processo industrial. Em seguida, mostraremos o modelo alternativo proposto por Nayebpour e Woodall (1993) e a derivação dos estimadores de máxima verossimilhança e de Bayes desenvolvida por Borges, Esteves e Wechsler (2005). Finalmente, apresentaremos uma nova solução para o problema de determinação do intervalo de inspeção ótimo sob a perspectiva da Teoria de Decisão Bayesiana. A última solução será ilustrada com alguns exemplos. 


\begin{abstract}
In this work, we present Taguchi's economic on line quality monitoring method for attributes. The aim of this method is to obtain the inspection interval that minimizes the expected cost per item produced in an industrial process. After that, we will consider the alternative method proposed by Nayebpour e Woodall (1993) and the derivation of the maximum likelihood and Bayes estimators developed by Borges, Esteves e Wechsler (2005). Finally, we will propose a new solution to the problem of inspection interval determination through Bayesian Decision Theory. This last solution will be implemented and its performance will be illustrated with a few examples.
\end{abstract}




\section{Sumário}

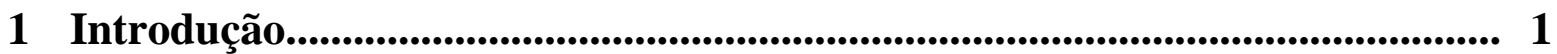

2 O Método de Taguchi On-Line para Atributos ................................................. 4

2.1 Método de Taguchi - Caso I ........................................................... 5

2.2 Método de Taguchi - Caso II ...................................................................... 10

2.3 Comentários Adicionais sobre o Método de Taguchi ..................................... 14

3 O Método Alternativo Proposto por Nayebpour e Woodall............................... 15

3.1 O Método de Nayebpour e Woodall - Caso I ................................................ 16

3.2 O Método de Nayebpour e Woodall - Caso II .......................................... 20

3.3 Estimação dos Parâmetros do Processo ......................................................... 23

4 Uma Abordagem Bayesiana....................................................................................... 32

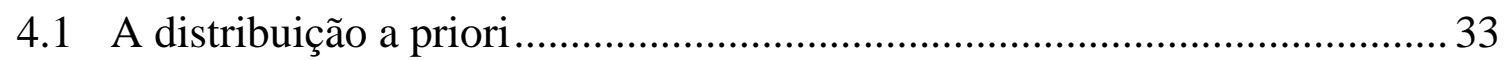

4.2 Inferência Bayesiana dos Parâmetros do Processo........................................... 34

4.3 Teoria de Decisão Bayesiana e a Determinação de m*................................... 36

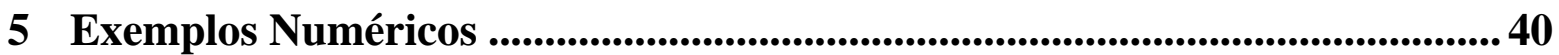

5.1 Programa para Obter m* pelo Método de Nayebpour e Woodall .................. 41

5.2 Programa para Obter m* pelo Método Bayesiano …….................................. 42

5.3 Programa para Obter m* por Simulação Estocástica ....................................... 44

5.4 Exemplos Numéricos................................................................................. 46 
6 Conclusão 53

A Cáculo da Experança da Expressão (4.10) 55

B Método de Nayebpour e Woodall 64

C Método de Borges, Esteves e Wechsler 65

$\begin{array}{ll}\text { D Abordagem Bayesiana } & 66\end{array}$

$\begin{array}{lll}\text { E Simulação Estocástica } & 68\end{array}$

Referências Bibliográficas $\quad 69$ 


\section{Capítulo 1}

\section{Introdução}

Os métodos estatísticos são fundamentais para a melhoria da qualidade de processos industriais, pois, através deles, é possível monitorar e controlar as características dos itens produzidos e detectar desvios significantes no processo produtivo que podem ter como conseqüência a produção de itens com características que não atendam às especificações pré-determinadas.

Uma das ferramentas do controle estatístico de processos (CEP) mais utilizadas com esse intuito é a carta de controle, criada por Shewhart em 1924. A operação das cartas de controle consiste em coletar periodicamente amostras de itens ou de conjuntos de itens produzidos e analisá-los de acordo com alguma característica de interesse. Os resultados observados em cada amostra são registrados graficamente em função do instante (tempo) de amostragem.

Na elaboração desse gráfico, além das estatísticas observadas nas amostras, são considerados alguns valores de referência: o limite inferior de controle, a linha central e o limite superior de controle. Os limites são utilizados para detectar se o processo está dentro ou fora de controle.

Existem vários tipos de cartas de controle que podem ser classificadas, de forma simplificada, em dois grandes grupos:

- Cartas de controle por variáveis - utilizadas quando a característica de interesse é medida em uma escala contínua.

Exemplos: a carta $\bar{X}$ utilizada para monitorar a média e a carta $R$ para monitorar a amplitude. 
- Cartas de controle por atributos - utilizadas quando a característica de interesse é binária ou uma contagem de características binárias.

Exemplos: a carta $p$ utilizada para monitorar a fração não conforme, a carta $n p$ para monitorar o número de itens não conformes e a carta $u$ para monitorar a quantidade de não conformidades por item.

Ao planejar o controle estatístico de um processo, é necessário definir: o tamanho das amostras, o intervalo entre as inspeções e os limites de controle (esses limites são, em geral, calculados utilizando um número pré-determinado de desvios padrões em relação à linha central). Vários autores sugeriram valores para esses elementos, entretanto, há um consenso apenas em relação ao uso de três desvios padrões para a determinação dos limites de controle. Para o tamanho das amostras e o intervalo entre as inspeções, em geral, os valores atribuídos variam de acordo com os critérios utilizados por cada autor.

Originalmente, a determinação desses elementos era baseada em aspectos puramente estatísticos, até que em 1956, Duncan propôs a integração dos aspectos estatísticos a um planejamento de processo baseado em critérios econômicos. Resumidamente, o objetivo de Duncan (1956) foi minimizar o custo total do processo, considerando todos os custos envolvidos em seu monitoramento e utilizando a carta $\bar{X}$.

Após o trabalho de Duncan (1956), vários autores sugeriram métodos econômicos para as cartas de controle. Especificamente para a carta $n p$, surgiram trabalhos a partir da década de 70, entre eles: Gibra (1978), Montgomery, Heikes e Mance (1975) e Williams, Looney e Peters (1985).

No final da década de 80, Taguchi, Elsayed e Hsiang propuseram um método econômico para monitorar variáveis de atributo em tempo real (on line). O objetivo desse método é determinar o intervalo ótimo de inspeção minimizando o custo por item, em um ciclo de produção.

O método de Taguchi é semelhante aos métodos econômicos desenvolvidos para a carta $n p$, porém Taguchi considera inspeções de apenas um item, ou seja, neste modelo o tamanho da amostra (n) é sempre igual a um. Outra diferença é que Taguchi não assume uma distribuição de probabilidade para o tempo necessário até o processo sair de controle.

Nesse trabalho, estudaremos o método econômico por atributos desenvolvido por Taguchi, o modelo alternativo proposto por Nayebpour e Woodall (1993), a derivação dos estimadores de máxima verossimilhança e de Bayes para o último modelo, desenvolvida por Borges, Esteves e Wechsler (2005) e, finalmente, abordaremos o problema de 
determinação do intervalo de inspeção ótimo sob a perspectiva da Teoria de Decisão Bayesiana. A última solução será ilustrada através de alguns exemplos.

No capítulo 2, estudaremos o método proposto por Taguchi et al (1989). Em seguida, dedicaremos o capítulo 3 ao método alternativo proposto por Nayebpour e Woodall (1993), no qual é considerada uma distribuição geométrica para o número de itens produzidos até o processo sair de controle. O processo de determinação do intervalo de inspeção ótimo baseado na Teoria de Decisão Bayesiana será mostrado no capítulo 4. No capítulo 5, será apresentado um algoritmo para simulação de conjuntos de dados e, através de alguns exemplos, serão ilustradas aplicações dos resultados do capítulo 4. 


\section{Capítulo 2}

\section{O Método de Taguchi On-Line para Atributos}

Considere um processo produtivo no qual é preciso manter a qualidade dos itens produzidos, isto é, estes devem satisfazer condições especificadas. Para garantir que o processo atenda a essas condições, os itens produzidos são inspecionados periodicamente e, caso sejam encontrados itens fora das especificações (itens não conforme), o processo é interrompido e ajustado. Após o ajuste, o processo volta imediatamente a operar nas condições iniciais e um novo ciclo começa.

Taguchi (1981, 1984, 1985) e Taguchi et al. (1989) desenvolveram um método econômico para auxiliar no controle de qualidade do processo por atributos descrito acima. O método consiste em minimizar o custo esperado por item através da determinação do intervalo de inspeção ótimo $\left(m^{*}\right)$. Para esse cálculo, é fixado o custo de inspeção, o custo de produção de um item não conforme e o custo de ajuste de processo.

O método considera dois casos. O primeiro, em que o processo, após sair de controle, passa a produzir apenas itens não conformes, será exibido na seção 2.1. Na seção 2.2, discutiremos o segundo caso, que ocorre quando o processo, após sair de controle, passa a produzir uma fração $(\theta)$ de itens não conformes, onde $0<\theta<1$.

Na seção 2.3, apresentaremos alguns comentários sobre o método proposto por Taguchi et al. (1989). 


\subsection{O Método de Taguchi - Caso I}

No modelo econômico proposto por Taguchi, os itens são inspecionados a cada $m$ unidades produzidas e, quando é encontrado o primeiro item não conforme, o processo é interrompido para realização de ajustes.

O objetivo do modelo é determinar o intervalo ótimo entre inspeções $\left(m^{*}\right)$, ou seja, o valor de $m$ que minimiza o custo esperado de produção de um item. Para isso, são considerados três custos:

- O custo de um item não conforme produzido $\left(C_{d}\right)$;

- O custo de amostragem e inspeção de um item $\left(C_{s}\right)$;

- O custo de ajuste do processo $\left(C_{a}\right)$.

Além disso, supõe-se que durante a inspeção de um item o processo produtivo continua. Isto é, se o item é considerado não conforme e o processo é interrompido para ajustes, teremos, em geral, um atraso de alguns itens produzidos entre o início da amostragem e a parada do processo: esse atraso é representado por $l$.

O número de itens produzidos no i-ésimo ciclo, ou seja, no intervalo entre os ajustes (sucessivos) dos ciclos $i-1$ e $i$, é representado por $u_{i}$, $i \in \mathrm{N}$, e $\bar{u}_{n}=\frac{\sum u_{i}}{n}$, denota o número médio de itens produzidos por ciclo.

A figura 2.1, extraída de Belem (2001), ilustra o processo produtivo com todos os fatores envolvidos.

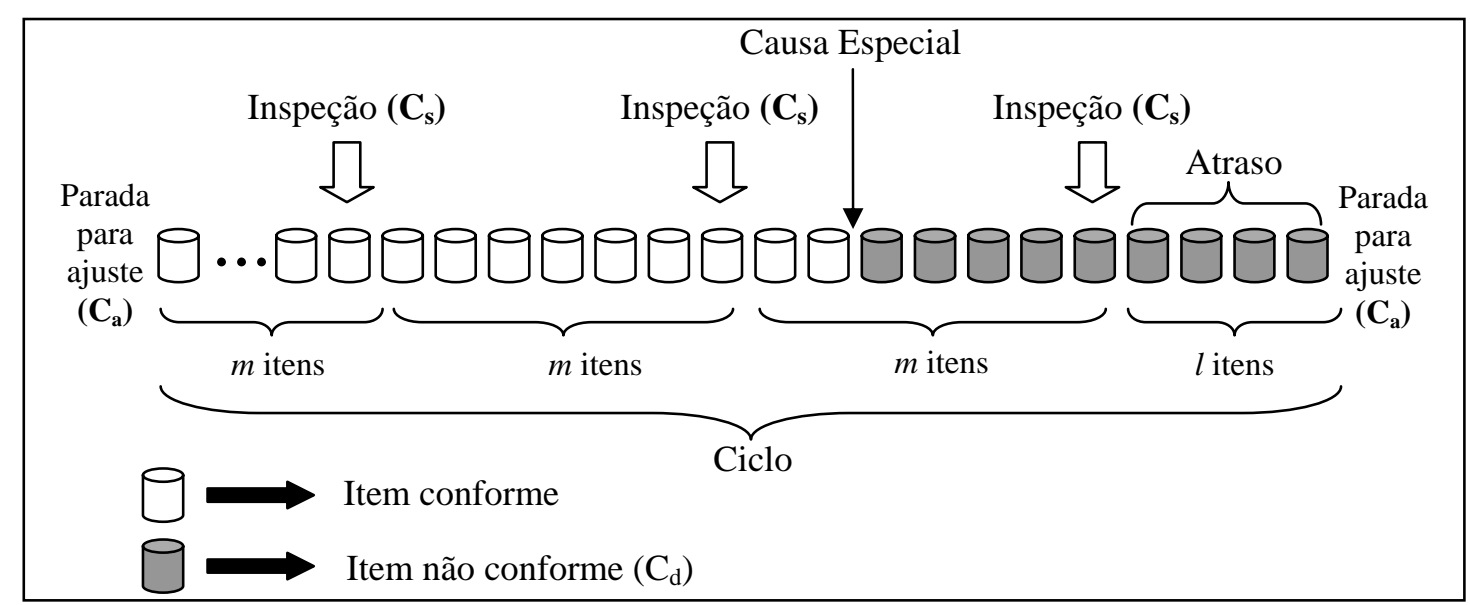

Figura 2.1: Ilustração de um ciclo com mudança da fração não conforme de 0 para 1 - Caso I. 
No caso I, a expressão para o custo esperado por item produzido em um ciclo é:

$$
L=\frac{C_{s}}{m}+\left(\frac{m+1}{2}+l\right) \frac{C_{d}}{\bar{u}}+\frac{C_{a}}{\bar{u}}
$$

Para justificar a primeira parcela, considere $\bar{k}$ o número médio de inspeções realizadas por ciclo até encontrar um item não conforme. Nesse caso, sendo $\bar{u}$ o tamanho médio de um ciclo, $\bar{u}=m \bar{k}$ (considerando $\bar{u}>>l$ ) e o custo de inspeção médio será:

$$
\frac{\bar{k} C_{s}}{\bar{u}}=\frac{C_{s}}{m}
$$

Como, para o caso I, após a mudança do processo, esse passa a produzir apenas itens não conformes, fica evidente que a mudança do processo ocorre entre as duas últimas inspeções do ciclo. Portanto, em cada ciclo são produzidos de $1 \mathrm{a} m$ itens não conformes. Considerando que a probabilidade de que qualquer um desses itens seja o primeiro não conforme é $\frac{1}{m}$ (conseqüentemente, as probabilidades de ter $1,2,3, \ldots$, ou $m$ itens não conformes são todas iguais a $\frac{1}{m}$ ) e calculando a esperança do número de itens não conformes, teremos, em média, $\frac{1}{m}+\frac{2}{m}+\ldots+\frac{m}{m}=\frac{m+1}{2}$ itens não conformes até o início da amostragem. Além disso, os $l$ itens produzidos até a parada do processo também serão não conformes. Com isso, o custo esperado de produção de itens não conformes no ciclo é $\left(\frac{m+1}{2}+l\right) \frac{C_{d}}{\bar{u}}$.

Assim, para obtermos o intervalo de inspeção ótimo, conforme feito por Taguchi et al. (1989), a expressão (2.1) será derivada em relação à $m$, igualada a zero e $m$ será determinado. Logo,

$$
L=\frac{C_{s}}{m}+\left(\frac{m+1}{2}+l\right) \frac{C_{d}}{\bar{u}}+\frac{C_{a}}{\bar{u}}
$$




$$
\frac{\partial L}{\partial m}=-\frac{C_{s}}{m^{2}}+\frac{C_{d}}{2 \bar{u}}=0
$$

Portanto,

$$
\begin{gathered}
m_{T 1}^{* 2} C_{d}=2 \bar{u} C_{s} \\
m_{T 1}^{*}=\sqrt{2 \bar{u} \frac{C_{s}}{C_{d}}}
\end{gathered}
$$

onde, $m_{T 1}^{*}$ denota o intervalo de inspeção ótimo de Taguchi para o caso I.

Sabemos que $m$ é um número natural e, portanto, a função do custo esperado não é diferenciável. Assim, após derivar a função, Taguchi et al. (1989) deveriam ter analisado os valores naturais mais próximos do valor obtido em (2.2).

Notamos ainda que ao derivar a equação (2.1), a dependência entre $\bar{u}$ e $m$ foi ignorada. Porém, essa dependência é evidente, pois $u_{i}=m k_{i}+l_{i}$, onde $k_{i}$ é o número de inspeções realizadas no i-esimo ciclo. Além disso, por não considerar essa dependência, o efeito de $l$ e $C_{a}$ foram desprezados e o valor de $m^{*}$ não será determinado com precisão, a não ser que $\bar{u}$ seja muito maior que $l(\bar{u}>>l)$ e $C_{d}$ seja muito maior que o custo de ajuste por item $\left(C_{d} \gg C_{a} / \bar{u}\right.$ ), de forma que a influência de $l$ e $C_{a}$ no custo seja desprezível.

Para os casos em que as condições acima não são satisfeitas, Taguchi et al (1989) recomendam o uso de uma expressão alternativa que substitui $\bar{u}$ por $\bar{u}+\frac{m}{2}$, argumentando que o número médio de itens não conformes produzidos no atraso é $\frac{m}{2}$. Essa argumentação é confusa, pois, por definição, $\bar{u}$ representa todos os itens produzidos (conformes e não conformes) e, nesse caso, estaríamos contando os itens não conformes do atraso duas vezes. Além disso, o número médio de itens não conforme produzidos já foi calculado e resultou em outra expressão. De qualquer forma, com a substituição obtemos a seguinte expressão: 


$$
L=\frac{C_{s}}{m}+\frac{m+1}{2} \frac{C_{d}}{\bar{u}+m / 2}+\frac{1}{\bar{u}+m / 2}\left(C_{a}+l C_{d}\right)
$$

Para resolver a equação acima, Taguchi et al. (1989) utilizaram a seguinte aproximação:

$$
\frac{1}{\bar{u}+m / 2} \approx \frac{1}{\bar{u}}\left(1-\frac{m}{2 \bar{u}}\right)
$$

Observe que para essa aproximação ser razoável, o valor de $m$ deverá ser pequeno quando comparado com o valor de $\bar{u}$.

Substituindo essa aproximação na expressão (2.3), derivando em relação à $m$, igualando o resultado a zero e isolando $m$, Taguchi et al. (1989) obtiveram o intervalo de inspeção ótimo:

$$
\begin{gathered}
L=\frac{C_{s}}{m}+\frac{m+1}{2 \bar{u}}\left(1-\frac{m}{2 \bar{u}}\right) C_{d}+\frac{1}{\bar{u}}\left(1-\frac{m}{2 \bar{u}}\right)\left(C_{a}+l C_{d}\right) \\
\frac{\partial L}{\partial m}=-\frac{C_{s}}{m^{2}}+\left(\frac{1}{2 \bar{u}}-\frac{2 m+1}{4 \bar{u}^{2}}\right) C_{d}+\frac{1}{2 \bar{u}^{2}}\left(C_{a}+l C_{d}\right)=0
\end{gathered}
$$

Portanto,

$$
\begin{gathered}
-\frac{C_{s}}{m_{T 1}^{* * 2}}+\frac{1}{2 \bar{u}} C_{d}+\frac{1}{2 \bar{u}^{-2}}\left(C_{a}+l C_{d}\right)=0 \\
-2 \bar{u}^{2} C_{s}+\bar{u} m_{T 1}^{* * 2} C_{d}+m_{T 1}^{* * 2}\left(C_{a}+l C_{d}\right)=0 \\
m_{T 1}^{* * 2}\left(C_{a}+l C_{d}+\bar{u} C_{d}\right)=2 \bar{u}^{2} C_{s} \\
m_{T 1}^{* *}=\sqrt{\frac{2 \bar{u}^{-2} C_{s}}{C_{a}+l C_{d}+\bar{u} C_{d}}} \\
m_{T 1}^{* *}=\sqrt{\frac{2 \bar{u} C_{s}}{C_{a} / \bar{u}+l C_{d} / \bar{u}+C_{d}}}
\end{gathered}
$$


Taguchi et al. (1989) argumentam que o termo $\frac{2 m+1}{4 \bar{u}^{-2}}$ é muito menor que o termo $\frac{1}{2 \bar{u}}$ e, por isso, é realizada uma aproximação, mantendo nos cálculos apenas o termo $\frac{1}{2 \bar{u}}$. Novamente, é importante observar que essa aproximação será razoável somente se $\bar{u}>>m$.

Para obter o resultado final, Taguchi et al. (1989) utilizam outra aproximação, partindo da suposição de que $C_{d} \gg>C_{a} / \bar{u}$ e $C_{d}>>l C_{d} / \bar{u}$. Assim,

$$
\begin{gathered}
\frac{1}{C_{a} / \bar{u}+I C_{d} / \bar{u}+C_{d}}=\frac{1}{\left(C_{d}-C_{a} / \bar{u}\right)\left(1-\frac{l C_{d} / \bar{u}}{C_{d}-C_{a} / \bar{u}}\right)} \\
\approx \frac{1}{\left(C_{d}-C_{a} / \bar{u}\right)}\left(1+\frac{l C_{d} / \bar{u}}{C_{d}-C_{a} / \bar{u}}\right) \\
\approx \\
\left(\frac{1}{\left(C_{d}-C_{a} / \bar{u}\right)}\left(1+\frac{l}{\bar{u}}\right)\right.
\end{gathered}
$$

Observe que a expressão (2.6) deveria ser uma alternativa para quando as condições $C_{d} \gg C_{a} / \bar{u}$ e $\bar{u} \gg>l$ não eram satisfeitas. Entretanto, para obter essa expressão, Taguchi et al. (1989) assumiram que essas condições eram válidas.

Para obter o intervalo de inspeção ótimo, Taguchi et al. (1989) substituem a expressão em (2.6) na equação (2.5):

$$
m_{T 1}^{* *}=\sqrt{\frac{2(\bar{u}+l) C_{s}}{C_{d}-C_{a} / \bar{u}}}
$$

Taguchi et al. (1989) argumentam que as diferenças entre as equações (2.2) e (2.7) diminuirão à medida que $C_{d}>C_{a} / \bar{u}$ e $\bar{u}>>l$. Quando ao menos uma dessas condições não é satisfeita, é recomendável utilizar a equação (2.7) à equação (2.2). 


\subsection{O Método de Taguchi - Caso II}

Taguchi et al. (1989) afirmam que quando o processo sai de controle e passa a produzir uma fração pequena de itens não conformes, as expressões (2.2) e (2.7) não são recomendadas, pois, com o uso destas, o intervalo ótimo de inspeção seria superestimado. Desta forma, eles apresentam uma equação alternativa para o caso em que $\theta<1$.

Para o caso II, o custo de um item não conforme produzido é desmembrado em duas partes:

- O custo de um item não conforme detectado $\left(C_{d}\right)$;

- O custo de um item não conforme não detectado e enviado para estágios posteriores do processo produtivo ou para o cliente $\left(\mathrm{C}_{D}\right)$, onde o custo $\mathrm{C}_{D}$ é especificado pelos autores como sendo muito maior que $\mathrm{C}_{d}\left(C_{D}>C_{d}\right)$.

A figura 2.2, extraída de Belem (2001), ilustra o processo produtivo com todos os fatores envolvidos.

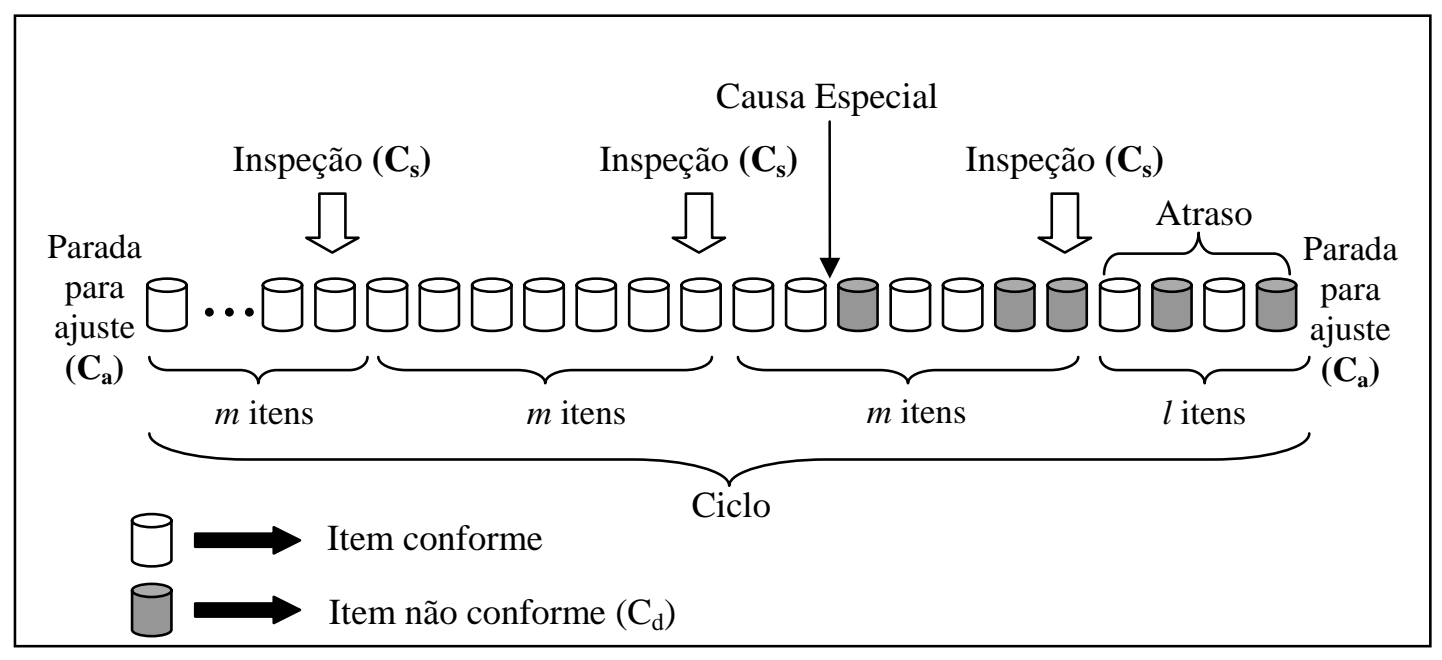

Figura 2.2: Ilustração de um ciclo com mudança da fração não conforme de 0 para $\theta(0<\theta<1)$.

Nesse caso, o processo após sair de controle passa a produzir uma fração $\theta$ de itens não conformes. Considerando uma distribuição uniforme para o primeiro item não conforme até a primeira inspeção após o processo sair de controle e utilizando um raciocínio análogo ao do caso $\mathrm{I}$, observamos que o número médio de itens não conformes produzidos até a primeira inspeção após o processo sair de controle será 
$\frac{(m+1) \theta}{2}$. Para os intervalos entre inspeções subseqüentes, considera-se que o número médio de itens não conformes é $m \theta$.

A probabilidade de encontrar um item não conforme na primeira inspeção após o processo sair de controle é $\theta$, a de encontrar apenas na segunda inspeção é $\theta(1-\theta)$, e, assim, sucessivamente. No caso da detecção de não conformidade na primeira inspeção, Taguchi et al. (1989) consideram que é feita inspeção retroativa nos $m$ itens produzidos até então, de modo que todos os itens não conformes serão detectados e considerados no custo $C_{d}$. Se um item não conforme é encontrado apenas na segunda inspeção, os itens não conformes produzidos antes da primeira inspeção são enviados para estágios posteriores interando o custo $C_{D}$. É feita ainda a inspeção retroativa nos $m$ itens produzidos entre a primeira e segunda inspeções e os itens não conformes encontrados nessa inspeção contribuirão para o custo $C_{d}$.

Considerando essas condições, Taguchi et al. (1989) obtiveram, conforme a tabela 2.1, o número médio de itens não conformes após o processo sair de controle em função do ponto de inspeção em que há a detecção de um item não conforme.

\begin{tabular}{cccc}
\hline $\begin{array}{c}\text { Ponto de } \\
\text { Inspeção }\end{array}$ & $\begin{array}{c}\text { Probabilidade de ter a } \\
\text { primeira detecção de um } \\
\text { item não conforme }\end{array}$ & $\begin{array}{c}\text { Número médio de } \\
\text { itens não conforme } \\
\text { detectados }\end{array}$ & $\begin{array}{c}\text { Número médio de itens } \\
\text { não conforme não } \\
\text { detectados }\end{array}$ \\
\hline$m$ & $\theta$ & $(m+1) \theta / 2$ & 0 \\
$2 m$ & $\theta(1-\theta)$ & $m \theta$ & $(m+1) \theta / 2$ \\
$3 m$ & $\theta(1-\theta)^{2}$ & $m \theta$ & $(m+1) \theta / 2+m \theta$ \\
$4 m$ & $\theta(1-\theta)^{3}$ & $m \theta$ & $(m+1) \theta / 2+2 m \theta$ \\
$\vdots$ & $\vdots$ & $\vdots$ & $\vdots$ \\
$i m$ & $\theta(1-\theta)^{i-1}$ & $m \theta$ & $(m+1) \theta / 2+(i-2) m \theta$ \\
$\vdots$ & $\vdots$ & $\vdots$ & $\vdots$ \\
\hline
\end{tabular}

Tabela 2.1: Número médio de itens não conformes em função do momento da amostragem do item não conforme, após o ponto de mudança. 
A partir da tabela 2.1, obtém-se o custo esperado total da produção dos itens não conformes, denominado $\mathrm{C}_{d+D}$ :

$$
\begin{gathered}
C_{d+D}=\left(\frac{(m+1) \theta^{2}}{2}+m \theta\left(\theta(1-\theta)+\theta(1-\theta)^{2}+\ldots\right)\right) C_{d}+ \\
+\left(\frac{(m+1) \theta}{2}\left(\theta(1-\theta)+\theta(1-\theta)^{2}+\ldots\right)+m \theta\left(\theta(1-\theta)^{2}+2 \theta(1-\theta)^{3}+\cdots\right)\right) C_{D} \\
C_{d+D}=\left(\frac{(m+1) \theta^{2}}{2}+m \theta(1-\theta)\right) C_{d}+\left(\frac{m+1}{2} \theta(1-\theta)+m(1-\theta)^{2}\right) C_{D}
\end{gathered}
$$

Baseados na expressão (2.8), Taguchi et al. (1989) argumentam que se $\theta=0$, a expressão é igual a $m C_{D}$. Portanto, assumindo-se que $\theta$ é próximo de zero, conclui-se que o custo de produção por item não conforme seria, aproximadamente, $m C_{D}$, que corresponde ao custo $\frac{(m+1) C_{d}}{2}$ do segundo termo da equação (2.1). Com isso, assumindo que $(m+1) \approx m$, Taguchi et al. (1989) substituíram $2 C_{D}$ por $C_{d}$ no segundo termo da equação (2.1) e obtiveram:

$$
\begin{aligned}
L & =\frac{C_{s}}{m}+\frac{(m+1) C_{d}}{2 \bar{u}}+\frac{C_{a}}{\bar{u}}+\frac{l C_{d}}{\bar{u}} \\
L & =\frac{C_{s}}{m}+(m+1) \frac{C_{D}}{\bar{u}}+\frac{C_{a}}{\bar{u}}+\frac{l C_{d}}{\bar{u}}
\end{aligned}
$$

Note que o último termo da expressão (2.9) considera que todos os itens produzidos durante o atraso são não conformes, porém essa situação não ocorre para o caso II, principalmente quando consideramos $\theta \approx 0$. De qualquer forma, considerando a expressão (2.9) e substituindo $2 C_{D}$ por $C_{d}$ na equação (2.7), o intervalo entre inspeções ótimo recomendado por Taguchi et al. (1989) é:

$$
m_{T 2}^{*}=\sqrt{\frac{2(\bar{u}+l) C_{s}}{2 C_{D}-C_{a} / \bar{u}}}
$$


Caso todas as unidades não conformes sejam detectadas por inspeção retrospectiva, não haverá custo de itens não conformes não detectados e enviados para estágios posteriores. Com isso, o custo $C_{d}$ substituíra o custo $C_{D}$ na equação (2.8) e, assim:

$$
C_{d+D}=\left(\frac{(m+1) \theta^{2}}{2}+m \theta(1-\theta)+\frac{m+1}{2} \theta(1-\theta)+m(1-\theta)^{2}\right) C_{d}
$$

Usando a aproximação $(m+1) \approx m$ na equação (2.11), temos:

$$
\begin{gathered}
C_{d+D}=\left(\frac{m \theta^{2}}{2}+\frac{3 m \theta(1-\theta)}{2}+m(1-\theta)^{2}\right) C_{d} \\
=\left(\frac{m \theta^{2}}{2}+\frac{3 m \theta}{2}-\frac{3 m \theta^{2}}{2}+m-2 m \theta+m \theta^{2}\right) C_{d} \\
C_{d+D}=m\left(1-\frac{\theta}{2}\right) C_{d}
\end{gathered}
$$

Portanto, o custo de realização da inspeção retrospectiva será máximo quando $\theta=0$ e mínimo quando $\theta=1$. Segundo Taguchi et al. (1989), o custo máximo por unidade não conforme seria $2 C_{d}$ e o mínimo seria $C_{d}$. O caso do custo mínimo já foi desenvolvido na seção 2.1. Para o custo máximo, substituindo $C_{D}$ por $C_{d}$ na equação (2.9), obtemos:

$$
L=\frac{C_{s}}{m}+(m+1) \frac{C_{d}}{\bar{u}}+\frac{C_{a}}{\bar{u}}+\frac{l C_{d}}{\bar{u}}
$$

Da mesma forma, o intervalo de inspeção ótimo será:

$$
m_{T 2}^{* *}=\sqrt{\frac{2(\bar{u}+l) C_{s}}{2 C_{d}-C_{a} / \bar{u}}}
$$


Para chegar às expressões das equações (2.10) e (2.14), Taguchi et al. (1989) assumiram que $\theta$ era conhecido e próximo de zero. Portanto, nos casos onde $\theta \geq 0,5$ eles recomendam o uso das equações obtidas para o Caso I.

\subsection{Comentários Adicionais sobre o Método de Taguchi}

No decorrer das seções 2.1 e 2.2, à medida que foi apresentado o método proposto por Taguchi et al. (1989), foram feitos alguns comentários a respeito das limitações desse método. Porém, há ainda alguns comentários adicionais para serem tratados nessa seção.

Na metodologia proposta por Taguchi et al. (1989), assume-se que a

probabilidade do processo sair de controle é igual a $\frac{1}{m}$ para qualquer momento do último intervalo entre inspeções. Os autores não apresentam um modelo probabilístico formal para o tempo de espera até o processo sair de controle. Além disso, os autores não assumem uma distribuição de probabilidade para o número de itens não conformes produzidos.

Além da metodologia não estar embasada em um modelo probabilístico, Taguchi et al. (1989) realizam algumas suposições e aproximações para obter as expressões do custo médio e do intervalo de inspeção ótimo. Com isso, há uma propagação de erros que aparentemente não é citada pelos autores. Dessa forma, os resultados obtidos por Taguchi et al. (1989) devem ser utilizados com cautela. 


\section{Capítulo 3}

\section{O Método Alternativo Proposto por Nayebpour e Woodall}

Nayebpour e Woodall (1993) examinaram o modelo proposto por Taguchi et al (1989) e propuseram um modelo alternativo para obter o custo esperado por item produzido, assumindo que o tempo necessário até o processo sair do controle tem distribuição geométrica de parâmetro $\rho, 0<\rho<1$.

Nayebpour e Woodall (1993) assumem que após cada ajuste do processo, iniciase um novo ciclo produtivo (período entre dois ajustes consecutivos do processo) com comportamento independente do ciclo anterior, porém com mesma estrutura probabilística.

Portanto, os autores consideram que os tempos de espera em cada ciclo até a detecção de um item não conforme (medidos em número de itens produzidos nos ciclos) são uma seqüência de variáveis aleatórias não negativas, independentes e identicamente distribuídas. Assim, o processo de contagem de número de itens produzidos nos ciclos pode ser considerado um processo de renovação (Ross, 1997).

A cada renovação (início de um ciclo), estão associados custos a esse processo de renovação: o custo de ajuste, os custos de inspeções realizadas no ciclo e dos itens não conformes produzidos no ciclo. Devido a essa característica do processo, poderemos considerar que a cada renovação há uma recompensa: nesse caso, uma recompensa negativa. Assumindo que essas recompensas são independentes e identicamente distribuídas, teremos um processo de renovação e recompensa (Ross, 1997). 
A representação do modelo através de um processo de renovação e recompensa permitiu a Nayebpour e Woodall (1993) obter uma expressão para o custo esperado por item produzido e encontrar o intervalo de inspeção que minimiza esse custo esperado.

Nayebpour e Woodall (1993) desenvolveram expressões para resolver o caso I e o caso II separadamente, assim como foi apresentado por Taguchi et al. (1989). Na seção 3.1, abordaremos o primeiro caso, em que o processo após sair de controle passa a produzir apenas itens não conformes. Na seção 3.2, teremos o segundo caso, em que após sair de controle o processo passa a produzir uma fração $(\theta)$ de itens não conformes, onde $0<\theta<1$.

Nas seções 3.1 e 3.2, mostraremos não só as expressões de custos, mas também os estimadores para os parâmetros dos modelos estatísticos propostos por Nayebpour e Woodall. Os autores encontraram tais estimadores pelo método dos momentos. Em particular, no caso II, em que o estimador de $\rho$ depende de um valor inicial de $m$, Nayebpour e Woodall não apresentam estimador para a fração não conforme $\theta$. Recentemente, Borges, Esteves e Wechsler (2005), de um modo formal, obtiveram o estimador de máxima verossimilhança para o par de parâmetros $(\theta, \rho)$. Esses resultados serão apresentados na seção 3.3.

\subsection{O Método de Nayebpour e Woodall - Caso I}

Nayebpour e Woodall desenvolveram um modelo para obtenção do intervalo de inspeção ótimo, assumindo que o número de itens produzidos até o processo sair de controle segue uma distribuição geométrica com parâmetro $\rho, 0<\rho<1$.

Na construção desse modelo, os autores consideraram:

- O custo ou recompensa por ciclo $(C)$;

- $\quad$ O número de itens produzidos por ciclo (T);

- O custo ou recompensa por item produzido $(L)$.

Representando o modelo através de um processo de renovação e recompensa, consideremos $C(i)$ o custo total até a conclusão do i-ésimo ciclo (calculado pela soma dos custos dos primeiros $i$ ciclos). Se $E(C)<\infty$ e $E(T)<\infty$, então, utilizando a lei forte dos grandes números, conclui-se que, com probabilidade $1, \lim _{i \rightarrow \infty} \frac{C(i)}{i}=\frac{E(C)}{E(T)}$. A prova do resultado acima pode ser encontrada em Ross (1997). 
Verificamos que a expressão $\lim _{i \rightarrow \infty} \frac{C(i)}{i}$ equivale ao custo esperado por item produzido $E(L)$. Desta forma, temos:

$$
E(L)=\frac{E(C)}{E(T)}
$$

Após encontrarem a expressão acima, Nayebpour e Woodall (1993) obtiveram as expressões para $E(T)$ e $E(C)$.

Conforme definido pelos autores, o número de itens produzidos até o processo sair de controle (ponto de mudança) segue uma distribuição geométrica com parâmetro $\rho$, que representa a probabilidade do processo passar a produzir itens não conformes. $\mathrm{O}$ número de inspeções até encontrar um item não conforme, segundo Nayebpour e Woodall, também seguirá uma distribuição geométrica. O parâmetro dessa distribuição geométrica será a probabilidade do processo sair de controle em uma determinada inspeção.

Para o caso I, o processo sairá de controle entre as duas últimas inspeções, ou seja, ao produzir os $m$ últimos itens. A probabilidade do processo não sair de controle na produção desses $m$ itens será $(1-\rho)^{m}$. Então, a probabilidade do processo sair de controle ao produzir um dos $m$ itens é $1-(1-\rho)^{m}$.

O número esperado de inspeções necessárias até encontrar um item não conforme será a esperança da variável aleatória com distribuição geométrica de parâmetro $1-(1-\rho)^{m}$, que é igual a $\frac{1}{1-(1-\rho)^{m}}$. Como as inspeções são realizadas a cada $m$ itens produzidos, o número esperado de itens produzidos até encontrar um item não conforme será $\frac{m}{1-(1-\rho)^{m}}$. Então, o número esperado de itens produzidos por ciclo será:

$$
E(T)=\frac{m}{1-(1-\rho)^{m}}+l
$$


Para obter $E(C)$, é necessário considerar os mesmos custos utilizados por Taguchi et al (1989): $C_{d}, C_{s}$ e $C_{a}$.

Para determinar $E(T)$, já vimos que o número de inspeções por ciclo até encontrar um item não conforme é $\frac{1}{1-(1-\rho)^{m}}$. Segundo Nayebpour e Woodall (1993), o custo esperado de inspeções por ciclo é:

$$
\left(\frac{1}{1-(1-\rho)^{m}}+\left\lfloor\frac{l}{m}\right\rfloor\right) C_{s} \text {, onde }\lfloor w\rfloor \text { representa o maior inteiro menor ou igual a } w \text {. }
$$

Observe que para obter a expressão acima, Nayebpour e Woodall (1993) consideram que as inspeções continuam a ser realizadas durante o atraso $(l)$.

O número de itens não conformes produzidos até encontrar um item não conforme é igual ao total de itens produzidos subtraindo os itens conformes. O número total esperado de itens produzidos é igual a $\frac{m}{1-(1-\rho)^{m}}$, calculado anteriormente. O número de itens conformes será o número de itens produzidos até o processo sair de controle menos um. Como essa variável aleatória tem distribuição geométrica com parâmetro $\rho$, o número de itens conformes produzidos é igual a $\frac{1}{\rho}-1$.

Como os itens produzidos durante o atraso são todos não conformes, o total de itens não conforme produzidos será $\frac{m}{1-(1-\rho)^{m}}-\frac{1}{\rho}+1+l$. Manipulando essa expressão, verifica-se que esta é igual ao coeficiente encontrado por Nayebpour e Woodall (1993) na expressão do custo esperado de um item não conforme produzido:

$$
\left(m-\frac{1-\rho}{\rho}+\frac{m(1-\rho)^{m}}{1-(1-\rho)^{m}}+l\right) C_{d}
$$


Calculando o limite de (3.4) quando $\rho \rightarrow 0$, através da aplicação da regra de L’Hôpital, conclui-se que a expressão converge para $\left(\frac{m+1}{2}+l\right) C_{d}$, que corresponde ao custo esperado de item não conforme em (2.1) obtido por Taguchi et al (1989).

Desta forma, o custo esperado por item produzido é:

$$
E(L)=\frac{\left(\frac{1}{1-(1-\rho)^{m}}+\left\lfloor\frac{l}{m}\right\rfloor\right) C_{s}+\left(m-\frac{1-\rho}{\rho}+\frac{m(1-\rho)^{m}}{1-(1-\rho)^{m}}+l\right) C_{d}+C_{a}}{\frac{m}{1-(1-\rho)^{m}}+l}
$$

A principal diferença entre as expressões do custo por item produzido obtida por Taguchi et al (1989) e por Nayebpour e Woodall (1993) é que o número médio de itens produzidos $(\bar{u})$ da expressão (2.1) foi substituído pelo número esperado de itens produzidos da expressão (3.2).

Belem (2001) apresentou uma correção para a expressão (3.5), retirando da expressão o termo $\left\lfloor\frac{l}{m}\right\rfloor$, pois, segundo a autora, durante o atraso não são realizadas inspeções.

No modelo de Nayebpour e Woodall (1993), $E(L)$ depende do parâmetro $\rho$ e, por isso, para obter o $m^{*}$, os autores devem, preliminarmente, estimar $\rho$, conforme mostramos a seguir.

Considerando $\eta$ a variável aleatória que representa o número de itens produzidos até o processo sair de controle, Nayebpour e Woodall (1993) assumiram que $\eta$ tem distribuição geométrica com parâmetro $\rho$ :

$$
P(\eta=n \mid \rho)=\rho(1-\rho)^{n-1}, n \in\{1,2, \ldots\}
$$

Seja $m_{c}$ o intervalo de inspeção inicial e considere $U_{1}, U_{2}, \ldots, U_{h}$ os números de itens produzidos em $h$ sucessivos ciclos produtivos ocorridos anteriormente, considerados independentes e identicamente distribuídos. Assim, sendo $E\left(U_{1}\right)=E\left(U_{2}\right)=\ldots E\left(U_{h}\right)=E(U)$, segue que: 


$$
E(U)=\frac{m_{c}}{1-(1-\rho)^{m_{c}}}+l
$$

Utilizando o método dos momentos com $\bar{U}=\frac{\sum_{i=1}^{h} U_{i}}{h}$ temos:

$$
\begin{gathered}
\bar{U}=\frac{m_{c}}{1-(1-\hat{\rho})^{m_{c}}}+l \\
\bar{U}-l-(\bar{U}-l)(1-\hat{\rho})^{m_{c}}=m_{c} \\
(1-\hat{\rho})^{m_{c}}=\frac{m_{c}+l-\bar{U}}{l-\bar{U}} \\
(1-\hat{\rho})=\left(1-\frac{m_{c}}{\bar{U}-l}\right)^{1 / m_{c}} \\
\hat{\rho}=1-\left(1-\frac{m_{c}}{\bar{U}-l}\right)^{1 / m_{c}}
\end{gathered}
$$

Utilizando valores históricos de $\bar{U}$, um valor inicial para o intervalo de inspeção, $m_{c}$, e substituindo este estimador na equação do custo esperado por item (3.5), os autores encontraram $m *$ através de busca direta.

\subsection{O Método de Nayebpour e Woodall - Caso II}

As expressões obtidas para o caso em que o processo passa a produzir itens não conformes com probabilidade $\theta(0<\theta<1)$ baseiam-se nos mesmos princípios da seção 3.1. A partir da expressão (3.1), obtém-se as expressões para $E(T)$ e $E(C)$.

O número esperado de itens produzidos em um ciclo será o número esperado de itens produzidos até a primeira inspeção após o processo sair do controle, já obtida em (3.2), somado ao número de itens não conformes produzidos até encontrar o primeiro item não conforme e ao atraso. 
A probabilidade do primeiro item não conforme ser encontrado na segunda inspeção após o ponto de mudança é $\theta(1-\theta)$, a de ser encontrado na terceira é $\theta(1-\theta)^{2}$ e a de ser encontrado, em geral, na i-ésima inspeção é $\theta(1-\theta)^{i-1}, i \geq 1$. Como entre cada par de inspeções consecutivas são produzidos $m$ itens, o número esperado de itens produzidos a partir da segunda inspeção após o ponto de mudança até a detecção de um item não conforme será:

$$
\sum_{i=1}^{\infty} i m \theta(1-\theta)^{i}=m \theta(1-\theta) \sum_{i=1}^{\infty} i(1-\theta)^{i-1}=\frac{m(1-\theta)}{\theta}
$$

Portanto, o número esperado de itens produzidos em um ciclo é:

$$
E(T)=\frac{m}{1-(1-\rho)^{m}}+\frac{m(1-\theta)}{\theta}+l
$$

Nayebpour e Woodall (1993) desmembram o custo de um item não conforme produzido em duas partes, da mesma forma que foi feito por Taguchi et al (1989).

Desse modo, obtiveram a seguinte expressão para o número esperado de itens não conformes produzidos até a primeira inspeção após o processo sair de controle:

$$
\left(m-\frac{1-\rho}{\rho}+\frac{m(1-\rho)^{m}}{1-(1-\rho)^{m}}+l\right) \theta
$$

A justificativa para essa expressão já foi apresentada na seção anterior, ao obter a expressão (3.4).

Com isso, o custo esperado dos itens não conformes produzidos para um ciclo do processo no caso II é:

$$
\begin{aligned}
E\left(C_{d+D}\right) & =\left(\left(m-\frac{1-\rho}{\rho}+\frac{m(1-\rho)^{m}}{1-(1-\rho)^{m}}\right) \theta^{2}+m \theta(1-\theta)+l \theta\right) C_{d} \\
& +\left(\left(m-\frac{1-\rho}{\rho}+\frac{m(1-\rho)^{m}}{1-(1-\rho)^{m}}\right) \theta(1-\theta)+m(1-\theta)^{2}\right) C_{D}
\end{aligned}
$$


Desta forma, se todos os itens no último intervalo entre inspeções tiverem inspeção retrospectiva, então o custo total esperado por ciclo será:

$$
E(C)=E\left(C_{d+D}\right)+\left(\frac{1}{1-(1-\rho)^{m}}+\frac{1-\theta}{\theta}+\left\lfloor\frac{l}{m}\right\rfloor\right) C_{s}+C_{a}
$$

Nayebpour e Woodall (1993) sugerem fazer a inspeção retrospectiva se $C_{s} \leq \theta C_{D}$. Caso a inspeção retrospectiva não seja feita, teremos uma redução da expressão (3.12) para:

$$
E\left(C_{d+D}\right)=\left(\left(m-\frac{1-\rho}{\rho}+\frac{m(1-\rho)^{m}}{1-(1-\rho)^{m}}\right) \theta+l \theta+m(1-\theta)\right) C_{D}
$$

O custo esperado por item é obtido através da equação (3.1) substituindo os valores de $E(C)$ da equação (3.13) e $E(T)$ da equação (3.10).

Belem (2001) apresentou correções às expressões (3.12) e (3.13). Na expressão (3.12), a autora acrescentou $(1-\theta)$ ao termo que multiplica $C_{d}$ e subtraiu $(1-\theta)$ ao termo que multiplica $C_{D}$. Na expressão (3.13), a autora argumenta que o termo $\left\lfloor\frac{l}{m}\right\rfloor$ deve ser retirado da expressão, pois, durante o atraso não há inspeção. Além disso, a autora sinalizou que caso a inspeção retrospectiva seja realizada, deve-se adicionar $l+m-1$ ao termo que multiplica o custo de inspeção.

Para um dado valor de $\theta$, o $\mathrm{m}^{*}$ foi encontrado da mesma forma que no caso I, obtendo o estimador pelo método dos momentos para $\rho$, conforme abaixo:

$$
\begin{gathered}
\bar{U}=E(U) \\
\bar{U}=\frac{m_{c}}{1-(1-\rho)^{m_{c}}}+\frac{m_{c}(1-\theta)}{\theta}+l \\
\theta\left[1-(1-\hat{\rho})^{m_{c}}\right](\bar{U}-l)=\theta m_{c}+\left[1-(1-\hat{\rho})^{m_{c}}\right] m_{c}(1-\theta)
\end{gathered}
$$




$$
\begin{aligned}
& {\left[1-(1-\hat{\rho})^{m_{c}}\right]\left(\theta \bar{U}-\theta l-m_{c}+\theta m_{c}\right)=\theta m_{c}} \\
& (1-\hat{\rho})^{m_{c}}=1-\frac{m_{c}}{\bar{U}-\left(l+\frac{m_{c}(1-\theta)}{\theta}\right)} \\
& \hat{\rho}=1-\left(1-\frac{m_{c}}{\bar{U}-\left(l+\frac{m_{c}(1-\theta)}{\theta}\right)}\right)^{1 / m_{c}}
\end{aligned}
$$

Utilizando valores históricos de $\bar{u}$ e $\theta$, um valor inicial para $m, m_{c}$, e substituindo este estimador na equação do custo esperado por item, os autores encontraram $m *$ através de busca direta.

O caso I é uma particularidade do caso II, bastando substituir $\theta=1$ nas expressões do caso II para obter as mesmas expressões do caso I.

Nayebpour e Woodall (1993) obtiveram apenas o estimador do parâmetro $\rho$ para encontrar o intervalo de inspeção ótimo. Porém, na expressão obtida pelos autores temos um par de parâmetros $(\theta, \rho)$ e, por isso, é recomendado obter o estimador para o par de parâmetros, e não apenas para $\rho$, conforme será apresentado na próxima seção.

\subsection{Estimação dos Parâmetros do Processo}

A expressão (3.13) do custo esperado por item depende do par de parâmetros $(\theta, \rho)$ que, em geral, são desconhecidos. Para encontrar o intervalo de inspeção que minimiza o custo esperado por item é preciso encontrar estimativas para esse par de parâmetros.

Nas seções anteriores, mostramos os estimadores para o parâmetro $\rho$ encontrados por Nayebpour e Woodall (1993) pelo método dos momentos, tanto para o caso I como para o caso II. No caso II, faz-se necessário utilizar valores históricos para determinar o parâmetro $\theta$, pois os autores não encontraram um estimador para esse parâmetro.

Borges, Esteves e Wechsler (2005) detalharam a análise do modelo proposto por Nayebpour e Woodall, analisando a função de verossimilhança (modelo estatístico) para 
os parâmetros $(\theta, \rho)$ gerada pelos dados (apresentada originalmente em Belem, 2001), desconsiderando os itens produzidos durante o atraso. Essa função permitiu encontrar o estimador de máxima verossimilhança para o par de parâmetros.

Definindo $\tau$ como a variável aleatória que representa número de inspeções necessárias até encontrar um item não conforme, obteremos a distribuição amostral de $\tau$ dados $\theta$ e $\rho$.

Para encontrar a distribuição $\tau \mid(\theta, \rho)$ precisamos saber entre quais inspeções o processo saiu de controle, passando a produzir itens não conformes. Considerando que essa mudança no processo ocorreu entre as inspeções dos itens $m j$ e $m(j+1)$, isto é, $m j<\eta \leq m(j+1)$, e utilizando probabilidades condicionais temos:

$$
\begin{gathered}
P(\tau=k \mid(\theta, \rho))=\sum_{j=0}^{k-1} \sum_{i=m j+1}^{m(j+1)} P(\tau=k \mid \eta=i, \theta, \rho) P(\eta=i \mid \theta, \rho) \\
P(\tau=k \mid(\theta, \rho))=\sum_{j=0}^{k-1} \theta(1-\theta)^{k-j-1} \sum_{i=m j+1}^{m(j+1)} P(\eta=i \mid \theta, \rho) \\
P(\tau=k \mid(\theta, \rho))=\sum_{j=0}^{k-1} \theta(1-\theta)^{k-j-1}[P(\eta>m j \mid(\theta, \rho))-P(\eta>m(j+1) \mid(\theta, \rho))] \\
P(\tau=k \mid(\theta, \rho))=\sum_{j=0}^{k-1} \theta(1-\theta)^{k-j-1}\left((1-\rho)^{m j}-(1-\rho)^{m(j+1)}\right) \\
P(\tau=k \mid(\theta, \rho))=\sum_{j=0}^{k-1} \theta(1-\theta)^{k-j-1}(1-\rho)^{m j}\left(1-(1-\rho)^{m}\right) \\
P(\tau=k \mid(\theta, \rho))=\theta\left(1-(1-\rho)^{m}\right)_{j=0}^{k-1}(1-\rho)^{m j}(1-\theta)^{k-1-j}
\end{gathered}
$$

Ou de forma análoga:

$$
P(\tau=k \mid(\theta, \rho))=\theta\left(1-(1-\rho)^{m}\right) \frac{(1-\rho)^{m k}-(1-\theta)^{k}}{(1-\rho)^{m}-(1-\theta)}
$$

Observe que a função de verossimilhança em (3.16), difere da obtida por Nayebpour e Woodall através da teoria da renovação. 
Para obter os estimadores de máxima verossimilhança para os parâmetros, utilizaremos uma reparametrização, considerando $\alpha=(1-\rho)^{m}$. Essa reparametrização será realizada para simplificar a notação e os cálculos. Após obter o estimador para $(\theta, \alpha)$, a propriedade de invariância dos estimadores de máxima verossimilhança (Casella e Berger, 2002) permitirá determinar o estimador para o vetor de parâmetros original $(\theta, \rho)$, bastando aplicar a transformação inversa.

Então, segundo a definição de estimador de máxima verossimilhança, precisamos obter $(\hat{\theta}, \hat{\alpha})$ de forma que:

$$
\begin{aligned}
& L_{k}(\hat{\theta}(k), \hat{\alpha}(k))=\max _{[0,1]^{2}} L_{k}(\theta, \alpha), \text { para cada } k \in\{1,2, \ldots\} \\
& \text { onde } L_{k}(\theta, \alpha)=P(\tau=k \mid(\theta, \alpha))=\theta(1-\alpha) \sum_{j=0}^{k-1} \alpha^{j}(1-\theta)^{k-1-j}
\end{aligned}
$$

Para $k=1$, a função de verossimilhança será $\theta(1-\alpha)$ e terá seu ponto de máximo quando $\theta=1$ e $\alpha=0$. Para $k>1$, encontraremos valores críticos da função $L_{k}(\theta, \alpha)$.

Primeiramente, derivando a função de verossimilhança em relação a $\theta$ e a $\alpha$, obtemos:

$$
\frac{\partial L_{k}(\theta, \alpha)}{\partial \theta}=\sum_{j=0}^{k-1} \alpha^{j}(1-\alpha)\left[(1-\theta)^{k-1-j}-\theta(k-1-j)(1-\theta)^{k-2-j}\right]
$$

e

$$
\frac{\partial L_{k}(\theta, \alpha)}{\partial \alpha}=\sum_{j=0}^{k-1} \theta(1-\theta)^{k-1-j}\left[j \alpha^{j-1}(1-\alpha)-\alpha^{j}\right]
$$

Para encontrar os pontos críticos da função (3.17) precisamos encontrar valores para os parâmetros $(\theta, \alpha)$ para os quais as derivadas são iguais à zero. A partir de (3.18), $(\theta, \alpha)$ deve satisfazer: 


$$
\begin{gathered}
\frac{\partial L_{k}(\theta, \alpha)}{\partial \theta}=\sum_{j=0}^{k-1} \alpha^{j}(1-\alpha)\left[(1-\theta)^{k-1-j}-\theta(k-1-j)(1-\theta)^{k-2-j}\right]=0 \\
\sum_{j=0}^{k-1} \alpha^{j}(1-\alpha)\left[(1-\theta)^{k-1-j}+\frac{(-k \theta+\theta+j \theta)(1-\theta)^{k-1-j}}{1-\theta}\right]=0 \\
\sum_{j=0}^{k-1} \alpha^{j}(1-\alpha)\left[\frac{(1-\theta)(1-\theta)^{k-1-j}+(-k \theta+\theta+\theta)(1-\theta)^{k-1-j}}{1-\theta}\right]=0 \\
(1-k \theta) \sum_{j=0}^{k-1} \alpha^{j}(1-\alpha)(1-\theta)^{k-1-j}=-\theta \sum_{j=0}^{k-1} j \alpha^{j}(1-\alpha)(1-\theta)^{k-1-j} \\
(1-k \theta) \theta(1-\alpha) \sum_{j=0}^{k-1} \alpha^{j}(1-\theta)^{k-1-j}=-\theta^{2}(1-\alpha) \sum_{j=0}^{k-1} j \alpha^{j}(1-\theta)^{k-1-j} \\
(1-k \theta) L_{k}(\theta, \alpha)=-\theta^{2}(1-\alpha) \sum_{j=0}^{k-1} j \alpha^{j}(1-\theta)^{k-1-j} \\
\sum_{j=0}^{k-1} j \alpha^{j}(1-\theta)^{k-1-j}=\frac{k \theta-1}{\theta^{2}(1-\alpha)} L_{k}(\theta, \alpha)
\end{gathered}
$$

Analogamente, a partir de (3.19), $(\theta, \alpha)$ deve satisfazer:

$$
\begin{gathered}
\frac{\partial L_{k}(\theta, \alpha)}{\partial \alpha}=\sum_{j=0}^{k-1} \theta(1-\theta)^{k-1-j}\left[j \alpha^{j-1}(1-\alpha)-\alpha^{j}\right]=0 \\
\sum_{j=0}^{k-1} \theta(1-\theta)^{k-1-j} j \alpha^{j-1}(1-\alpha)=\sum_{j=0}^{k-1} \theta(1-\theta)^{k-1-j} \alpha^{j} \\
\frac{\theta(1-\alpha)}{\alpha} \sum_{j=0}^{k-1} j \alpha^{j}(1-\theta)^{k-1-j}=\theta \sum_{j=0}^{k-1} \alpha^{j}(1-\theta)^{k-1-j} \\
\frac{\theta(1-\alpha)^{2}}{\alpha} \sum_{j=0}^{k-1} j \alpha^{j}(1-\theta)^{k-1-j}=\theta(1-\alpha) \sum_{j=0}^{k-1} \alpha^{j}(1-\theta)^{k-1-j} \\
\frac{\theta(1-\alpha)^{2}}{\alpha} \sum_{j=0}^{k-1} j \alpha^{j}(1-\theta)^{k-1-j}=L_{k}(\theta, \alpha) \\
\sum_{j=0}^{k-1} j \alpha^{j}(1-\theta)^{k-1-j}=\frac{\alpha}{\theta(1-\alpha)^{2}} L_{k}(\theta, \alpha)
\end{gathered}
$$


A partir das expressões (3.20) e (3.21), verificamos que a estimativa de máxima verossimilhança quando $\tau=k,\left(\hat{\theta}_{0}, \hat{\alpha}_{0}\right)$, deve satisfazer:

$$
\begin{gathered}
\frac{\hat{\alpha}_{0}}{\hat{\theta}_{0}\left(1-\hat{\alpha}_{0}\right)^{2}}=\frac{k \hat{\theta}_{0}-1}{\hat{\theta}_{0}^{2}\left(1-\hat{\alpha}_{0}\right)} \\
\hat{\alpha}_{0} \hat{\theta}_{0}=k \hat{\theta}_{0}-1-k \hat{\theta}_{0} \hat{\alpha}_{00}+\hat{\alpha}_{0} \\
\hat{\alpha}_{0}\left(\hat{\theta}_{0}+k \hat{\theta}_{0}-1\right)=k \hat{\theta}_{0}-1 \\
\hat{\alpha}_{0}=\frac{k \hat{\theta}_{0}-1}{(k+1) \hat{\theta}_{0}-1}
\end{gathered}
$$

A relação 3.22 é representada no gráfico abaixo:

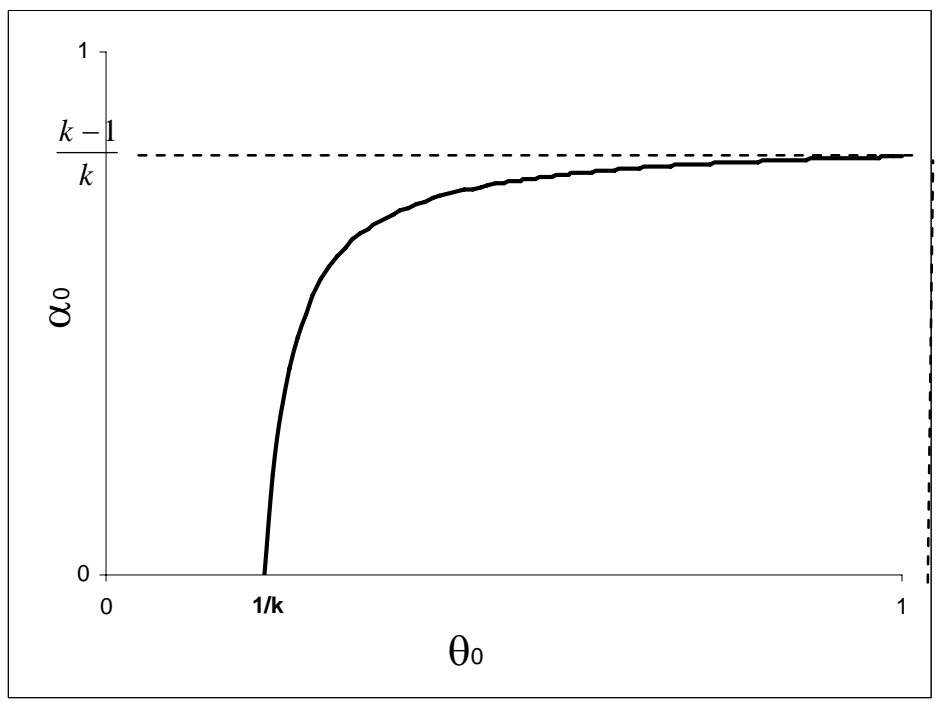

Figura 3.1: Gráfico de $\alpha_{0}$ em função de $\theta_{0}$.

Utilizando a expressão (3.22), podemos reescrever a função de verossimilhança em (3.17) como função de $\theta$ (substituindo $\alpha$ por $\alpha(\theta)$ ):

$$
L_{k}(\theta)=\theta\left(1-\frac{k \theta-1}{(k+1) \theta-1}\right) \sum_{j=0}^{k-1}\left[\frac{k \theta-1}{(k+1) \theta-1}\right]^{j}(1-\theta)^{k-1-j}
$$




$$
\begin{aligned}
& L_{k}(\theta)=\frac{\theta^{2}}{(k+1) \theta-1} \sum_{j=0}^{k-1}\left[\frac{k \theta-1}{(k+1) \theta-1}\right]^{j}(1-\theta)^{k-1-j} \\
& L_{k}(\theta)=\frac{\theta^{2}(1-\theta)^{k-1}}{[(k+1) \theta-1]} \sum_{j=0}^{k-1}\left[\frac{k \theta-1}{(\{k+1\} \theta-1)(1-\theta)}\right]^{j}
\end{aligned}
$$

Quando $\frac{k \theta-1}{[(k+1) \theta-1](1-\theta)} \neq 1$, podemos reescrever a expressão (3.23) da seguinte forma:

$$
\begin{gathered}
L_{k}(\theta)=\frac{\theta^{2}(1-\theta)^{k-1}}{[(k+1) \theta-1]} \frac{1-\left[\frac{k \theta-1}{(\{k+1\} \theta-1)(1-\theta)}\right]^{k}}{1-\left[\frac{k \theta-1}{(\{k+1\} \theta-1)(1-\theta)}\right]} \\
L_{k}(\theta)=\frac{\theta^{2}(1-\theta)^{k-1}}{[(k+1) \theta-1]} \frac{(1-\theta)^{k}-\left[\frac{k \theta-1}{(\{k+1\} \theta-1)}\right]^{k}}{(1-\theta)-\left[\frac{k \theta-1}{(\{k+1\} \theta-1)}\right]}\left(\frac{1}{(1-\theta)^{k-1}}\right) \\
L_{k}(\theta)=\frac{\theta^{2}}{[(k+1) \theta-1]} \frac{(1-\theta)^{k}-\left[\frac{k \theta-1}{(\{k+1\} \theta-1)}\right]^{k}}{(1-\theta)-\left[\frac{k \theta-1}{(\{k+1\} \theta-1)}\right]} \\
L_{k}(\theta)=\frac{(1-\theta)^{k}[(k+1) \theta-1]^{k}-(k \theta-1)^{k}}{[(k+1) \theta-1} \frac{(k+1) \theta-1}{(1-\theta)[(k+1) \theta-1]-k \theta+1} \\
L_{k}(\theta)=\frac{\theta^{2}(1-\theta)^{k}[(k+1) \theta-1]^{k}-\theta^{2}(k \theta-1)^{k}}{[(k+1) \theta-1]^{k}\left(\theta-k \theta^{2}-\theta^{2}\right)}
\end{gathered}
$$

A função de verossimilhança em (3.17) é tal que $L_{k}(\theta, \alpha)=L_{k}(1-\alpha, 1-\theta)$ e, portanto, podemos restringir o estudo da função em (3.24) ao intervalo $\left(\frac{1}{k}, \frac{2}{k+1}\right)$.

Derivando a expressão (3.24) em relação a $\theta$, obtemos: 


$$
\frac{\partial L_{k}(\theta)}{\partial \theta}=\frac{\left.\left[k(k+1) \theta^{2}-2(k+1) \theta+2\right] k \theta-1\right]^{k-1}}{[2-(k+1) \theta]^{2}[(k+1) \theta-1]^{k+1}}\left[\frac{(1-\theta)^{k-1}(\{k+1\} \theta-1)^{k+1}}{(k \theta-1)^{k-1}}-1\right]
$$

Como $\quad k(k+1) \theta^{2}-2(k+1) \theta+2>0 \quad$ para $\left.\quad \forall \theta \in\right] / k, 2 /(k+1)[, \quad$ então $\partial L_{k}(\theta) / \partial \theta>0$ se, e somente se, $h(\theta)=\left[\frac{(1-\theta)^{k-1}(\{k+1\} \theta-1)^{k+1}}{(k \theta-1)^{k-1}}-1\right]>0$.

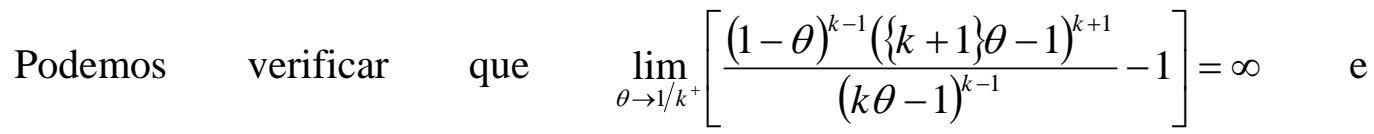
$\lim _{\theta \rightarrow 2 /(k+1)^{-}}\left[\frac{(1-\theta)^{k-1}(\{k+1\} \theta-1)^{k+1}}{(k \theta-1)^{k-1}}-1\right]=0$. Além disso, derivando em relação a $\theta$ a função $h(\theta)=\left[\frac{(1-\theta)^{k-1}(\{k+1\} \theta-1)^{k+1}}{(k \theta-1)^{k-1}}-1\right]$, obtemos $\frac{-k(k \theta-1)^{k-2}(1-\theta)^{k-2}[(k+1) \theta-1]^{k}[(k+1) \theta-2]^{2}}{(k \theta-1)^{2 k+1}}<0, \quad$ para $\quad$ todo $\left.\theta \in\right] / k, 2 /(k+1)[$. Portanto, temos que a função $h$ é estritamente decrescente no intervalo $] / k, 2 /(k+1)[$ e, assim, $h(\theta)>0$ nesse intervalo (e, conseqüentemente, $\partial L_{k}(\theta) / \partial \theta>0$ ). Portanto, concluímos que $L_{k}(\theta)$ atinge o ponto de máximo em $\theta_{0}=2 /(k+1)$.

Substituindo o valor encontrado para $\theta_{0}$ na expressão (3.22), obtemos $\alpha_{0}=(k-1) /(k+1)$. Então, pela análise acima, o ponto de máximo interior para $L_{k}(\theta, \alpha)$ é $(2 /(k+1),(k-1) /(k+1))$.

Avaliando os extremos do espaço paramétrico, notamos que, para os casos em que $\theta=0$ ou $\alpha=1$, a função de verossimilhança vale zero, enquanto para os casos em que $\theta=1$ e $\alpha=0$ (adotando a convenção $0^{0}=1$ ), utilizando a expressão (3.22), concluímos que $L_{k}(\theta, \alpha)$ terá ponto de máximo $(1,(k-1) / k)$ e $(1 / k, 0)$, respectivamente.

Para comparar os pontos de máximo nos extremos do espaço paramétrico com o ponto de máximo interior de $[0,1]^{2}$ e verificar qual é o ponto de máximo da função, substituiremos os valores na expressão (3.17). Realizando a substituição para os pontos 
$(1,(k-1) / k)$ e $(1 / k, 0)$, obtemos $L_{k}(\theta, \alpha)=(k-1)^{k-1} / k^{k}$. Considerando o ponto $(2 /(k+1),(k-1) /(k+1))$ obtemos $L_{k}(\theta, \alpha)=4 k(k-1)^{k-1} /(k+1)^{k+1}$.

Compararemos então o valor da função de verossimilhança no ponto $(1 / k, 0)$, que é $(k-1)^{k-1} / k^{k} \quad$ com o valor no ponto $(2 /(k+1),(k-1) /(k+1))$, que é $4 k(k-1)^{k-1} /(k+1)^{k+1}$. Para isso, dividiremos uma expressão pela outra e verificaremos se o resultado é maior (ou menor) que 1. Efetuando a divisão, obtemos a expressão $\frac{1}{4}\left(\frac{k+1}{k}\right)^{k+1}$ que é uma função decrescente em relação a $k$ (para provar que a função é decrescente, basta derivar a expressão em relação a $k$, obtendo $\frac{(k(k+1))^{k+1}}{4 k^{2 k+2}}\left(\ln \left(\frac{k+1}{k}\right)-\frac{1}{k}\right)$ e observar que a derivada é maior que zero para qualquer $k>1)$.

Como a função é decrescente, seu maior valor será quando $k=1$. Nesse caso, $\frac{1}{4}\left(\frac{k+1}{k}\right)^{k+1}=1$. Caso contrário, $\frac{1}{4}\left(\frac{k+1}{k}\right)^{k+1}<1$. Portanto, para qualquer $k>1$, $4 k(k-1)^{k-1} /(k+1)^{k+1} \quad$ é $\quad$ maior que $\quad(k-1)^{k-1} / k^{k}$. Logo, $\quad(\hat{\theta}(k), \hat{\alpha}(k))=$ $(2 /(k+1),(k-1) /(k+1)), \quad k \geq 1, \quad$ ou, $\quad$ de forma equivalente, $(\hat{\theta}, \hat{\alpha})=(2 /(\tau+1),(\tau-1) /(\tau+1))$ é o estimador de máxima verossimilhança para $(\theta, \alpha)$. Pela propriedade da invariância dos estimadores de máxima verossimilhança e considerando um valor inicial para $m, m_{c}$, temos:

$$
(\hat{\theta}, \hat{\rho})=\left(\frac{2}{\tau+1}, 1-\left(\frac{\tau-1}{\tau+1}\right)^{\frac{1}{m_{c}}}\right)
$$

Quando há informações disponíveis de $n$ ciclos produtivos independentes (com mesmo intervalo entre inspeções $m$ ), isto é, quando tivermos observado as quantidades $\tau_{1}, \tau_{2}, \ldots \tau_{n}$ independentes e identicamente distribuídas dados $\theta$ e $\rho$, o estimador de 
máxima verossimilhança será $\left(\frac{2}{\bar{\tau}+1}, 1-\left(\frac{\bar{\tau}-1}{\bar{\tau}+1}\right)^{\frac{1}{m}}\right)$, onde $\bar{\tau}$ é a média de $\tau_{1}, \tau_{2}, \ldots \tau_{n}$. A prova é análoga a obtida para o caso de apenas um ciclo (em que temos apenas uma observação de $\tau$ ).

É possível provar que o estimador de momentos para $\rho$ encontrado por Nayebpour e Woodall é igual ao encontrado por Borges, Esteves e Wechsler. Para isso, basta substituir o valor de $\bar{u}=m \bar{\tau}+l$ e de $\hat{\theta}$ na expressão (3.15).

Observamos que para os estimadores de máxima verossimilhança obtidos, $\theta$ será sempre maior que $\rho$ para $m>1$ e no caso em que $m=1$, teremos $\theta=\rho$. Por isso, não é aconselhável utilizar esses estimadores quando sabe-se previamente que $\theta<\rho$.

Além disso, o estimador de máxima verossimilhança é obtido utilizando-se apenas a função de verossimilhança como fonte de informação sobre o vetor de parâmetros desconhecidos, não incorporando a opinião de um especialista sobre tais parâmetros. No próximo capítulo, apresentaremos uma abordagem bayesiana para o problema. Em geral, a abordagem bayesiana permite utilizar a informação (opinião) que um especialista tem (a priori) dos parâmetros do processo. Em particular, no nosso problema, a abordagem bayesiana pode contemplar situações em que sabe-se, de antemão, que $\theta<\rho$. 


\section{Capítulo 4}

\section{Uma Abordagem Bayesiana}

$\mathrm{Na}$ abordagem bayesiana, o grau de incerteza que um indivíduo atribui para cada valor (ou intervalo de valores) que os parâmetros podem assumir é traduzido formalmente através de uma distribuição de probabilidade, denominada como distribuição a priori.

A partir da distribuição a priori e da informação fornecidas pelos dados a respeito dos parâmetros, expressa pela função de verossimilhança, um bayesiano obtêm, utilizando o Teorema de Bayes, a expressão de incerteza atualizada que contem toda informação disponível sobre os parâmetros e é denominada distribuição a posteriori.

Por depender da informação (incerteza) a priori e da verossimilhança, a abordagem bayesiana torna-se mais flexível e abrangente que a abordagem clássica, podendo ser aplicada a uma série de processos produtivos. Em particular, no cenário que estamos estudando, a abordagem bayesiana incorpora o conhecimento que um especialista possui dos parâmetros do processo sob investigação.

Para os casos em que o conhecimento a priori é escasso, a abordagem bayesiana pode ainda ser aplicada, pois esta permite assumir uma distribuição a priori não informativa, conforme apresentado por Paulino, Turkman e Murteira (2003). Esses autores argumentam que uso de distribuição a priori não informativa também é importante por permitir uma comparação adequada com os resultados da inferência clássica que considera apenas a informação amostral (Bernardo e Smith, 1994).

A seção 4.1 será dedicada a apresentar sumariamente a escolha da distribuição a priori para o nosso caso. 
No final do capítulo anterior, mostramos o estimador de máxima verossimilhança do parâmetro $(\theta, \rho)$ encontrado por Borges, Esteves e Wechsler (2005). Esse é um dos métodos utilizados em inferência clássica para obtenção de estimadores para os parâmetros. Para os bayesianos, a melhor inferência para os parâmetros é a distribuição a posteriori. Essa inferência também foi apresentada por Borges, Esteves e Wechsler (2005). Mostraremos esse resultado na seção 4.2.

Os conceitos básicos da teoria de decisão bayesiana serão introduzidos na seção 4.3, onde obteremos uma regra de decisão para encontrar o intervalo de inspeção ótimo.

\subsection{A distribuição a priori}

A distribuição a priori deve representar, numericamente, o conhecimento que o especialista tem do parâmetro de interesse, antes da coleta dos dados. Para isso, é necessário quantificar o conhecimento (subjetivo) que o especialista possui do parâmetro, descrevendo-o através de uma distribuição de probabilidade.

Para expressar o conhecimento a priori sobre um parâmetro de interesse em uma distribuição de probabilidade a priori, costuma-se utilizar uma família de distribuições conjugadas, conforme exposto por Paulino, Turkman e Murteira (2003). Os parâmetros da distribuição a priori são denominados hiperparâmetros para distingui-los do parâmetro de interesse.

Usualmente, costuma-se utilizar como distribuição a priori modelos que conduzam a uma distribuição a posteriori com mesma forma funcional. Desta forma, segundo Paulino, Turkman e Murteira (2003) a atualização do conhecimento que se tem do parâmetro depende apenas de uma mudança (conjugação) nos hiperparâmetros.

No nosso caso, temos que trabalhar com a função de verossimilhança (3.16) e uma distribuição conjunta para o parâmetro $(\theta, \rho)$. A escolha de uma classe de distribuições que seja fechada por amostragem, considerando a verossimilhança (3.16), não é tão simples como em outros exemplos.

Para simplificar, assumiremos que $\theta$ e $\rho$ são independentes e adotaremos distribuições Beta como priori para $\theta$ e $\rho$. Assim,

$$
f(\theta, \rho)=\frac{1}{\beta\left(a_{1}, b_{1}\right)} \theta^{a_{1}-1}(1-\theta)^{b_{1}-1} \frac{1}{\beta\left(a_{2}, b_{2}\right)} \rho^{a_{2}-1}(1-\rho)^{b_{2}-1}
$$


onde $\beta(a, b)$ representa a função beta, e $a_{1}, b_{1}>0$ e $a_{2}, b_{2}>0$ são os pares de parâmetros das distribuições marginais Beta a priori dos parâmetros $\theta$ e $\rho$ $\left(\theta \sim \operatorname{Beta}\left(a_{1}, b_{1}\right)\right.$ e $\left.\rho \sim \operatorname{Beta}\left(a_{2}, b_{2}\right)\right)$.

A suposição de independência dos parâmetros além de simplificar a expressão da distribuição a priori e, conseqüentemente, da distribuição a posteriori, também é uma suposição razoável nesse cenário de inferência.

\subsection{Inferência Bayesiana dos Parâmetros do Processo}

Utilizando a distribuição a priori apresentada na seção anterior e a função de verossimilhança obtida na seção 3.3, determinaremos a distribuição a posteriori para $(\theta, \rho)$, representada por $f(\theta, \rho \mid \tau=k)$. Tal distribuição é obtida considerando $l=0$ (inexistência de atraso).

Aplicando o Teorema de Bayes, temos:

$$
f_{k}(\theta, \rho)=f(\theta, \rho \mid \tau=k) \propto L_{k}(\theta, \rho) f(\theta, \rho)
$$

Substituindo a expressão (3.16) da função de verossimilhança $L_{k}(\theta, \rho)$ e a distribuição a priori (4.1) na equação (4.2), temos:

$$
f(\theta, \rho \mid \tau=k) \propto\left(1-(1-\rho)^{m}\right) \sum_{j=0}^{k-1}(1-\rho)^{m j+b_{2}-1}(1-\theta)^{k-1-j+b_{1}-1} \frac{1}{\beta\left(a_{2}, b_{2}\right)} \rho^{a_{2}-1} \frac{1}{\beta\left(a_{1}, b_{1}\right)} \theta^{a_{1}}
$$

Então,

$$
f(\theta, \rho \mid \tau=k)=C^{-1}\left(1-(1-\rho)^{m}\right) \sum_{j=0}^{k-1} \theta^{a_{1}}(1-\theta)^{k-1-j+b_{1}-1} \rho^{a_{2}-1}(1-\rho)^{m+b_{2}-1}
$$

onde

$$
C=C\left(m, k, a_{1}, b_{1}, a_{2}, b_{2}\right)=\sum_{j=0}^{k-1} \beta\left(a_{1}+1, b_{1}+k-1-j\right)\left[\beta\left(a_{2}, b_{2}+m j\right)-\beta\left(a_{2}, b_{2}+(j+1) m\right)\right]
$$


Escrevendo $\quad 1-(1-\rho)^{m}=\rho\left(1+(1-\rho)+(1-\rho)^{2}+\ldots+(1-\rho)^{m-1}\right), \quad$ podemos observar que $f(\theta, \rho \mid \tau=k)$ é uma densidade que corresponde a uma mistura de Betas independentes.

Integrando a distribuição a posteriori conjunta para $(\theta, \rho)$ em função de cada parâmetro, obtemos as marginais para $\theta$ e $\rho$. Assim:

$$
f(\theta \mid \tau=k)=C^{-1} \sum_{j=0}^{k-1}\left[\beta\left(a_{2}, b_{2}+m j\right)-\beta\left(a_{2}, b_{2}+(j+1) m\right)\right] \theta^{a_{1}}(1-\theta)^{b_{1}+k-j-2}
$$

Observamos, na expressão acima, que a distribuição de $\theta \mid \tau=k$ é uma mistura de $k$ distribuições Beta com parâmetros $a_{1}+1$ e $b_{1}+k-j-1, j=0,1, \ldots, k-1$, ponderada por $\quad p_{j}=C^{-1}\left[\beta\left(a_{2}, b_{2}+m j\right)-\beta\left(a_{2}, b_{2}+(j+1) m\right)\right] \beta\left(a_{1}+1, b_{1}+k-j-1\right)$, respectivamente.

Utilizando uma notação simplificada, podemos escrever:

$$
\theta \mid \tau=k \sim \sum_{j=0}^{k-1} p_{j} \operatorname{Beta}\left(a_{1}+1, b_{1}+k-j-1\right)
$$

De forma análoga, concluímos que:

$$
\begin{gathered}
\rho \mid \tau=k \sim \sum_{j=0}^{k-1} u_{j} \operatorname{Beta}\left(a_{2}, b_{2}+m j\right)-\sum_{j=0}^{k-1} v_{j} \operatorname{Beta}\left(a_{2}, b_{2}+(j+1) m\right) \\
\text { onde: } u_{j}=C^{-1} \beta\left(a_{1}+1, b_{1}+k-j-1\right) \beta\left(a_{2}, b_{2}+m j\right) \mathrm{e} \\
v_{j}=C^{-1} \beta\left(a_{1}+1, b_{1}+k-j-1\right) \beta\left(a_{2}, b_{2}+(j+1) m\right)
\end{gathered}
$$

Ou seja, a distribuição de $\rho \mid \tau=k$ é a diferença de duas misturas de $k$ distribuições Beta (ou ainda, uma mistura de $k m$ distribuições Beta). 
Notamos, pelas expressões (4.5), (4.6) e (4.7), que apesar de utilizarmos distribuições a priori independentes para $\theta$ e $\rho$, essa independência não é mantida na distribuição a posteriori, exceto no caso em que $\tau=1$.

A partir das distribuições a posteriori (marginais e conjunta), é possível obter as médias, medianas e modas a posteriori para cada parâmetro. Entretanto, a melhor inferência que podemos apresentar para o par de parâmetros $(\theta, \rho)$ é a distribuição $a$ posteriori.

Além de estimar os parâmetros do processo, a abordagem bayesiana permite fazer inferências para o momento em que o processo sai de controle (ponto de mudança), quantidade que também é de interesse e que não figura no modelo estatístico proposto por Nayebpour e Woodall (1993). E, como nas outras abordagens, é possível encontrar o intervalo de inspeção ótimo $\left(m^{*}\right)$. Esse assunto será abordado na próxima seção.

\subsection{Teoria de Decisão Bayesiana e a Determinação de $m^{*}$}

Segundo DeGroot (1970), os elementos fundamentais na especificação de um problema de decisão são:

- O espaço de todos os valores que o parâmetro desconhecido (w) pode assumir, denominado espaço paramétrico $(\Omega)$;

- A distribuição de probabilidade do parâmetro, $P(w)$;

- O espaço de todas as decisões possíveis $(d)$, chamado de espaço de decisões $(D)$;

- A função de perda que assume valores reais e está definida no espaço $\Omega \times D$. Para cada ponto $(w, d) \in \Omega \times D$, o número $L(w, d)$ representa a perda quando o valor do parâmetro $W$ é we a decisão escolhida é $d$.

Em geral, para cada $d \in D$, a perda $L(., d)$ é uma função mensurável (variável aleatória) no espaço $\Omega$.

Para qualquer decisão $d \in D$, a perda esperada, ou risco, $\pi(P, d)$, é dada por:

$$
\pi(P, d)=\int_{\Omega} L(w, d) d P(w)
$$


Em geral, são consideradas apenas as decisões $d \in D$ para as quais a integral na equação (4.8) é finita. As decisões $d$ para as quais essa suposição não é verdadeira podem ser desconsideradas, pois não serão decisões ótimas. Para encontrar a melhor decisão, é necessário escolher, se possível, a decisão $(d)$ para a qual o risco $\pi(P, d)$ é minimizado. Em muitos problemas, é conveniente trabalhar com funções de perda não negativas e, para isso, é necessário realizar uma mudança na função perda original, conforme mostrado por DeGroot (1970).

Especificamos, na seqüência, os elementos do problema de decisão para o nosso cenário. Vamos considerar que, a cada ciclo, um novo problema de escolha do intervalo ótimo entre inspeções deve ser resolvido. Assim, para o nosso modelo, temos:

- O espaço paramétrico, $\Omega=[0,1]^{2} \times\{0,1\}^{\infty}$. Um elemento típico de $\Omega$ é $w=\left(\theta, \rho, x_{1}, x_{2}, ..\right)$, onde $x_{i}=0$, indica se o i-ésimo item produzido é conforme e $x_{i}=1$, se o i-ésimo item produzido é não conforme;

- A distribuição de probabilidade do parâmetro, $P$, é especificada da seguinte forma: definimos uma distribuição conjunta para $(\theta, \rho)$ e consideramos que $X_{i} /(\theta, \rho)$ está distribuído segundo o modelo proposto por Nayebpour e Woodall (1993), para $i=1$, 2, 3,... . Vamos considerar que a distribuição conjunta de $(\theta, \rho)$ muda de ciclo para ciclo, pois há um aprendizado sobre esses parâmetros a cada ciclo. Assim, para o primeiro ciclo, resolvemos o problema de decisão considerando a priori (4.1) para $(\theta, \rho)$. Para o segundo ciclo, o problema de decisão é resolvido considerando a posteriori para $(\theta, \rho)$ dada a informação do primeiro ciclo (expressão 4.4) e, assim, sucessivamente;

- $\mathrm{O}$ espaço de decisões, é $\mathrm{D}=\mathrm{N} \backslash\{0\}=\{1,2,3, \ldots\}$;

- A função de perda $L(w, m)=L\left(\left(\theta, \rho, x_{1}, x_{2}, ..\right), m\right)$ que representa o custo por item produzido em um ciclo.

Nesse caso, a função de perda (desconsiderando o atraso) é dada por:

$$
L(w, m)=\frac{C_{a}+\tau(w, m) C_{s}+C_{d} \sum_{i=1}^{m \tau(w, m)} x_{i}}{m \tau(w, m)}
$$


Na expressão (4.9) $\tau(w, m)=\inf \left\{k \in \mathrm{N}: x_{k m}=1\right\}$ e para os pontos $(w, m)$ tais que $\tau(w, m)=\infty$, consideramos $L(w, m)=\infty$.

A partir da função de perda e da distribuição para $(\theta, \rho)$ especificada, podemos calcular o risco $\pi(P, m)$, que é a perda esperada da decisão $m \in \mathrm{N} \backslash\{0\}$. Minimizando o risco em $m$, encontraremos uma decisão ótima, ou seja, o $m^{*}$ que minimiza o custo esperado por item em um ciclo. Essa forma utilizada para a obtenção da decisão de Bayes é denominada de forma extensiva (DeGroot, 1970).

Portanto, para achar o intervalo de inspeção ótimo, precisamos calcular, para cada valor de $m$,

$$
\begin{gathered}
\pi(P, m)=E(L)=E(E(L \mid \theta, \rho)) \\
=\int_{0}^{1} \int_{0}^{1} E(L \mid \theta, \rho) f(\theta, \rho) d \theta d \rho
\end{gathered}
$$

O risco $\pi(P, m)$ para cada decisão $m \in D$ é dado por (4.11). A demonstração desse resultado está apresentada no Apêndice A.

$$
\begin{gathered}
\pi(P, m)=\int_{0}^{1} \int_{0}^{1} E(L \mid \theta, \rho) f(\theta, \rho) d \theta d \rho \\
=\int_{0}^{1} \int_{0}^{1}\left\{\frac{C_{s}}{m}+\frac{C_{a}+C_{d}}{m} \frac{\theta\left(1-(1-\rho)^{m}\right)}{(1-\rho)^{m}-(1-\theta)} \ln \left(\frac{\theta}{1-(1-\rho)^{m}}\right)+\frac{\theta(m-1) C_{d}}{m}+\right. \\
-\frac{\theta C_{d}}{m}\left(\frac{\theta\left(1-m \rho-(1-\rho)^{m}\right)}{\rho\left((1-\theta)-(1-\rho)^{m}\right)} \ln \left(\frac{1-(1-\rho)^{m}}{\theta}\right)+\frac{\theta(1-\theta)(m-1)\left(1-(1-\rho)^{m}\right)}{\left((1-\theta)-(1-\rho)^{m}\right)^{2}} \ln \left(\frac{1-(1-\rho)^{m}}{\theta}\right)+\right. \\
\left.\left.-\frac{\theta(m-1)(1-\rho)^{m}}{(1-\theta)-(1-\rho)^{m}}\right)\right\} f(\theta, \rho) d \theta d \rho
\end{gathered}
$$

Resolvendo a equação (4.11) para o caso em que $m=1$ temos $E(L \mid \theta, \rho)=C_{s}+\left(C_{a}+C_{d}\right) \frac{\theta \rho}{\rho-\theta} \ln \left(\frac{\rho}{\theta}\right)$ e quando $m \rightarrow \infty$ temos $E(L \mid \theta, \rho)=\theta C_{d}$. Avaliando o resultado para o caso em que $m \rightarrow \infty$, verificamos que este é intuitivo, ou 
seja, caso não sejam feitas inspeções teremos apenas o custo de um item não conforme produzido que será multiplicado pela fração de não conformidade.

Para encontrarmos o intervalo de inspeção ótimo $\left(m^{*}\right)$, precisamos substituir a esperança de $L$ dado $\theta, \rho$ na expressão (4.10) da perda esperada a posteriori dado $\tau=k$, resolver as integrais e minimizar o resultado em função de $m$.

Não é possível resolver as integrais da expressão da perda esperada a posteriori analiticamente, sendo necessário recorrer a métodos numéricos para obter um resultado aproximado. No próximo capítulo, apresentaremos o algoritmo utilizado para resolver a integral e obter o $m^{*}$. 


\section{Capítulo 5}

\section{Exemplos Numéricos}

Nos capítulos anteriores, apresentamos alguns métodos para encontrar o intervalo de inspeção ótimo para o procedimento on line de controle para atributos: o método de Taguchi (capítulo 2), o método de Nayebpour e Woodall (capítulo 3) e o método bayesiano (capítulo 4). Nesse capítulo, apresentaremos programas para encontrar o $\mathrm{m}^{*}$ a partir de cada método e aplicaremos esses resultados a alguns exemplos numéricos.

Para avaliar o desempenho dos métodos, elaboramos um programa baseado em técnicas de simulação que permitirá reproduzir a situação real e calcular o $m$ *

Os programas foram desenvolvidos para o caso II, em que o processo sai de controle e passa a produzir uma fração $\theta$ de itens não conformes $(0<\theta<1)$. Consideramos que não é feita a inspeção retrospectiva e que não existe atraso, ou seja, nos programas não teremos a variável $l(l=0)$.

Para o método de Taguchi é possível encontrar o intervalo de inspeção ótima de forma direta através das equações apresentadas no capítulo 1, por isso não será necessário desenvolver um programa para esse método. O programa para o método de Nayebpour e Woodall e o programa utilizando os estimadores de máxima verossimilhança para os parâmetros obtidos por Borges, Esteves e Wechsler (2005) são apresentados na seção 5.1. A seção 5.2 dedica-se ao programa para o método bayesiano baseado em Teoria da Decisão.

Na seção 5.3, descreveremos o programa de simulação estocástica utilizado para reproduzir o comportamento das variáveis aleatórias envolvidas em um processo produtivo 
de itens que em determinado momento sai de controle e passa a produzir uma fração de itens não conformes. Após a simulação desse ambiente, teremos o número de itens produzidos e o número de itens não conformes por ciclo e poderemos então calcular o custo médio por item por intervalo de inspeção e encontrar o intervalo de inspeção ótimo.

A partir desses programas, poderemos avaliar, para alguns exemplos, os resultados obtidos em cada método e os resultados simulados. Esse tópico será abordado na seção 5.4.

\subsection{Programa para Obter $m^{*}$ pelo Método de Nayebpour e Woodall}

Aplicando o método desenvolvido por Nayebpour e Woodall (1993) através das expressões apresentadas na seção 3.2, encontramos o intervalo de inspeção ótimo utilizando o seguinte programa:

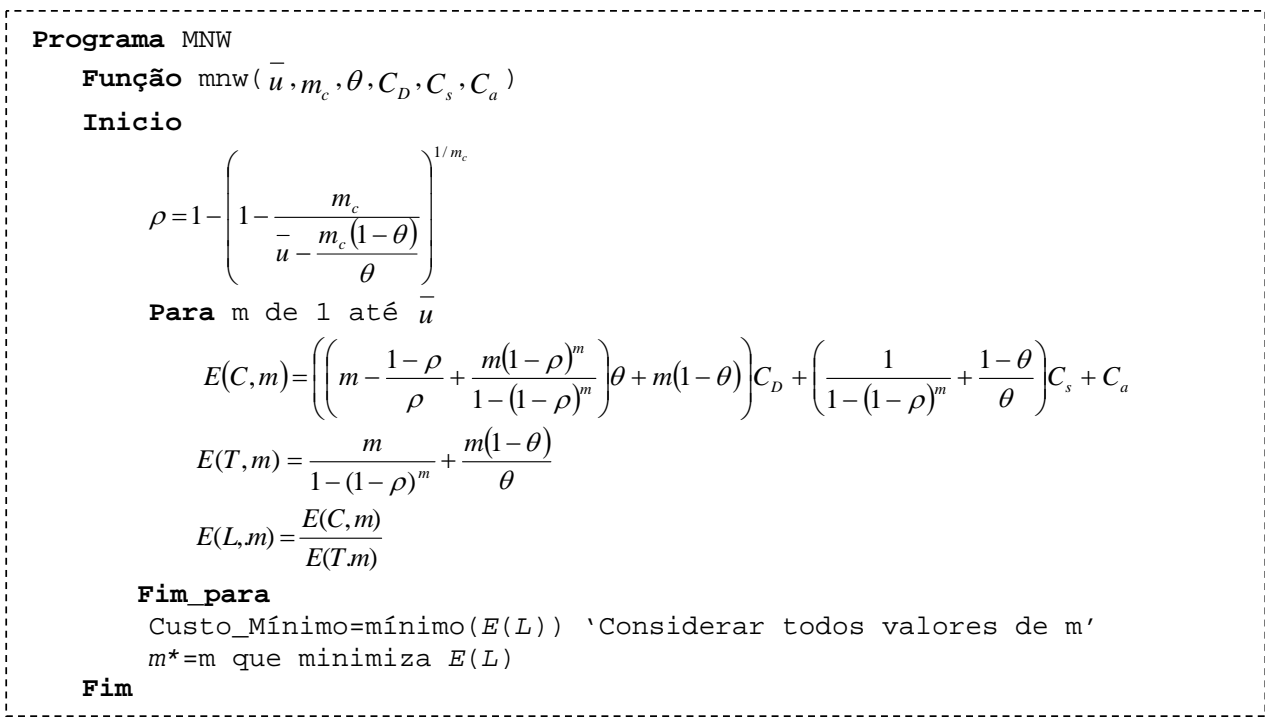
Woodall.

Figura 5.1: Programa em pseudocódigo para obter $0 \mathrm{~m}^{*}$ pelo método de Nayebpour e

O programa mostrado na figura 5.1 foi elaborado de acordo com o método Nayebpour e Woodall (1993) e, por isso, é necessário definir o parâmetro $\theta$ para encontrar o intervalo de inspeção ótimo. Desenvolvemos um segundo programa que não necessita da definição do parâmetro $\theta$. Nesse programa, são utilizadas as estimativas de máxima verossimilhança (EMV) para os parâmetros $\theta$ e $\rho$ obtidas por Borges, Esteves e Wechsler (2005), conforme exibido na seção 3.3. 


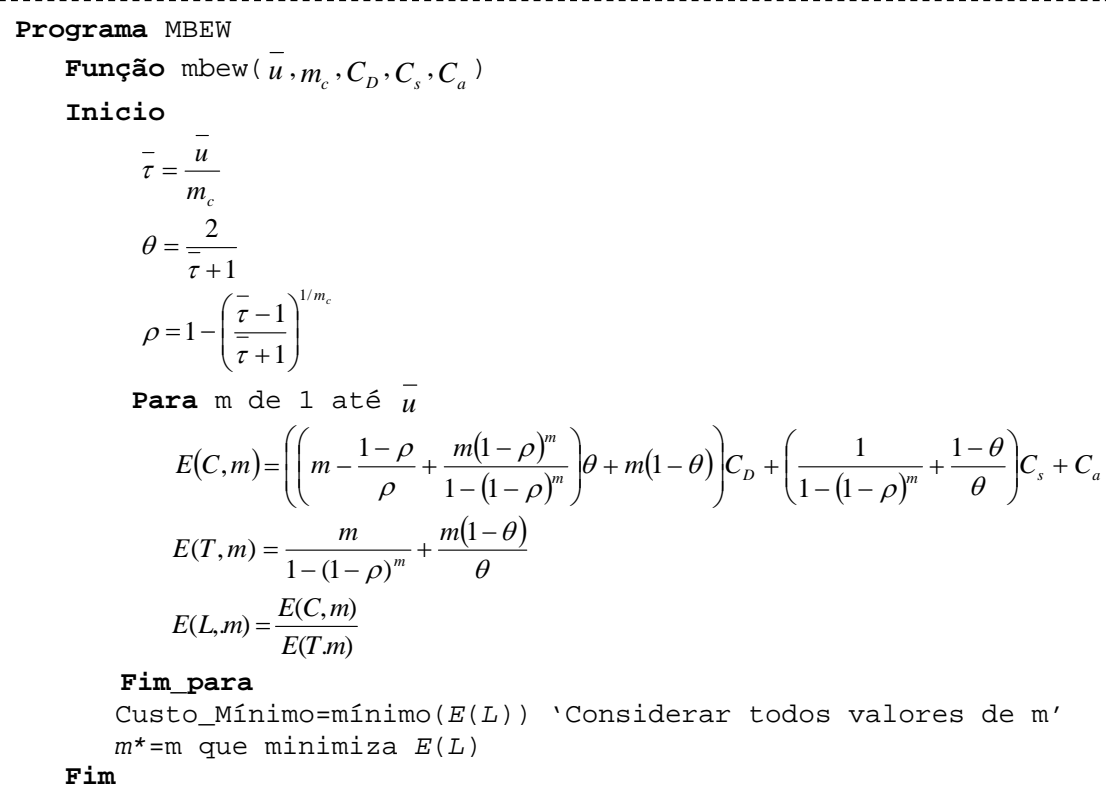

Figura 5.2: Programa em pseudocódigo para obter o $m^{*}$ utilizando os EMV para os parâmetros.

Nesse programa assumimos que o valor de $m_{c}$ é o mesmo em todos os ciclos, utilizados para calcular $\bar{u}$. Com isso, a seguinte igualdade torna-se válida: $\bar{\tau}=\overline{\left(\frac{u}{m}\right)}=\frac{\bar{u}}{m_{c}}$, conforme utilizamos no programa.

Os dois programas apresentados nessa seção foram implementados no software Matlab R12. O código fonte do primeiro programa encontram-se no Apêndice B e o do segundo no Apêndice C.

\subsection{Programa para Obter $\boldsymbol{m}^{*}$ pelo Método Bayesiano}

Aplicando o método bayesiano baseado em Teoria da Decisão e utilizando a expressão (4.11) apresentada na seção 4.3, desenvolvemos um programa para obter o intervalo de inspeção ótimo. 


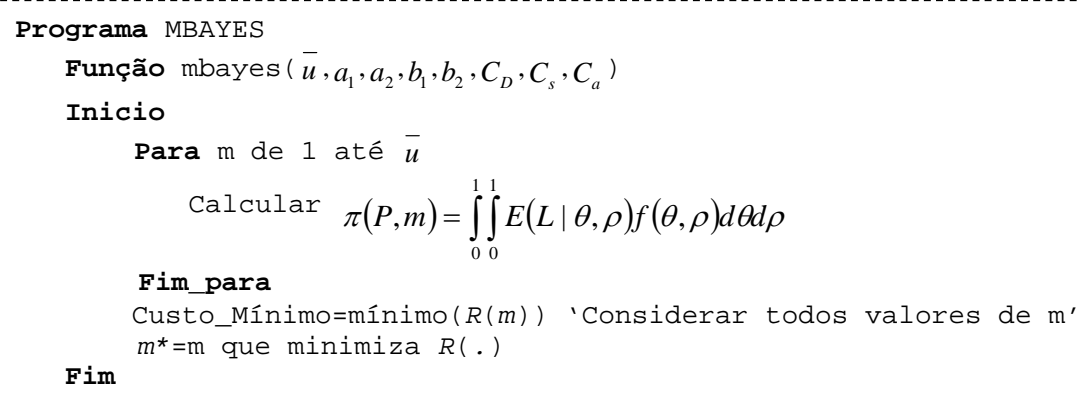

Figura 5.3: Programa em pseudocódigo para obter 0 m* utilizando a abordagem bayesiana.

Apesar de o programa ser aparentemente simples, a resolução da integral acima é complexa. Se fosse possível resolver a integral analiticamente, bastaria encontrar o mínimo da função resultante para obter o intervalo de inspeção ótimo e, nesse caso, não seria necessária a implementação deste pseudocódigo.

Entretanto, não foi possível encontrar uma solução analítica para a integral e tivemos que recorrer a métodos de integração numérica. A princípio, tentamos resolver numericamente sem assumir valores para $m$. Porém, como o processamento tornou-se muito custoso, tivemos que resolver a expressão para cada valor de $m$ e, para limitar o intervalo de valores de $m$, foi necessário definir previamente um valor máximo para $m$, por exemplo, $\bar{u}$.

Para implementar o pseudocódigo solucionamos separadamente a integral de cada termo da expressão (4.22).

Observe que não é preciso utilizar o método de integração numérica para todos os termos da expressão. O primeiro termo pode ser integrado de forma trivial. Por se tratar de uma constante, $\frac{C_{s}}{m}$ pode ser isolado, restando integrar $f(\theta, \rho)$ que resultará em 1 , já que estamos integrando uma função de densidade de probabilidade para todos os valores assumidos pelo parâmetro.

O terceiro termo também pode ser resolvido analiticamente, isolando a constante $\frac{(m-1) C_{d}}{m}$ e resolvendo a integral:

$$
\int_{0}^{1} \int_{0}^{1} \frac{1}{\beta\left(a_{2}, b_{2}\right)} \rho^{a_{2}-1}(1-\rho)^{b_{2}-1} \frac{1}{\beta\left(a_{1}, b_{1}\right)} \theta^{a_{1}}(1-\theta)^{b_{1}-1} d \rho d \theta
$$




$$
\begin{gathered}
=\int_{0}^{1} \frac{1}{\beta\left(a_{1}, b_{1}\right)} \theta^{a_{1}}(1-\theta)^{b_{1}-1} d \theta \\
=\frac{\beta\left(a_{1}+1, b_{1}\right)}{\beta\left(a_{1}, b_{1}\right)} \\
=\frac{\Gamma\left(a_{1}+1\right) \Gamma\left(b_{1}\right)}{\Gamma\left(a_{1}+b_{1}+1\right)} \frac{\Gamma\left(a_{1}+b_{1}\right)}{\Gamma\left(a_{1}\right) \Gamma\left(b_{1}\right)} \\
=\frac{a_{1} !\left(b_{1}-1\right) !}{\left(a_{1}+b_{1}\right) !} \frac{\left(a_{1}+b_{1}-1\right) !}{\left(a_{1}-1\right) !\left(b_{1}-1\right) !}=\frac{a_{1}}{a_{1}+b_{1}}
\end{gathered}
$$

Portanto, será necessário integrar numericamente o segundo e o quarto termo, que será dividido em três partes.

Para resolver a integral numericamente fizemos testes com o método do trapezóide (Mathews, 1992), o método da quadratura de Simpson, o método da quadratura de Lobato (Gander e Gautschi, 2000) e o método de Monte Carlo (Gamerman, 1997). Os quatro métodos apresentam problemas de convergência dependendo do valor de $m$ e dos hiperparâmetros. Porém, o método de Monte Carlo teve um desempenho superior aos demais métodos e, por isso, o pseudocódigo foi implementado utilizando esse método.

O programa apresentado nessa seção foi implementado no software Matlab R12 e o código fonte encontra-se no Apêndice D.

\subsection{Programa para Obter $m$ * por Simulação Estocástica}

Nos capítulos anteriores, vimos que no inicio do ciclo, o processo está sob controle, não produzindo itens não conformes, e que o parâmetro $\rho$ representa a probabilidade do processo passar a produzir itens não conformes.

Para simular os itens produzidos até o processo sair de controle (ponto de mudança) geraremos números aleatórios uniformemente distribuídos entre zero e um. A cada número gerado que for maior que $\rho$ consideraremos que foi produzido um item conforme e que o processo está sob controle e quando for gerado um número menor que $\rho$ iremos considerar que o processo saiu de controle (ponto de mudança), podendo produzir itens não conformes. 
No caso II, após o processo sair de controle, ele passa a produzir itens não conforme com probabilidade $\theta$. Então, considerando $X_{i}$ uma variável que indica se o $i$ ésimo item produzido após o processo sair de controle é não conforme, teremos $X_{i} \mid \theta \sim \operatorname{Bernoulli}(\theta)$.

Para simular o período após a saída de controle do processo, geraremos números aleatórios com distribuição de $\operatorname{Bernoulli}(\theta)$. Quando o número gerado for igual a um consideraremos que o item produzido é não conforme, se for gerado o número zero, o item será não conforme.

Dadas as condições acima desenvolvemos implementamos o seguinte programa em pseudocódigo:

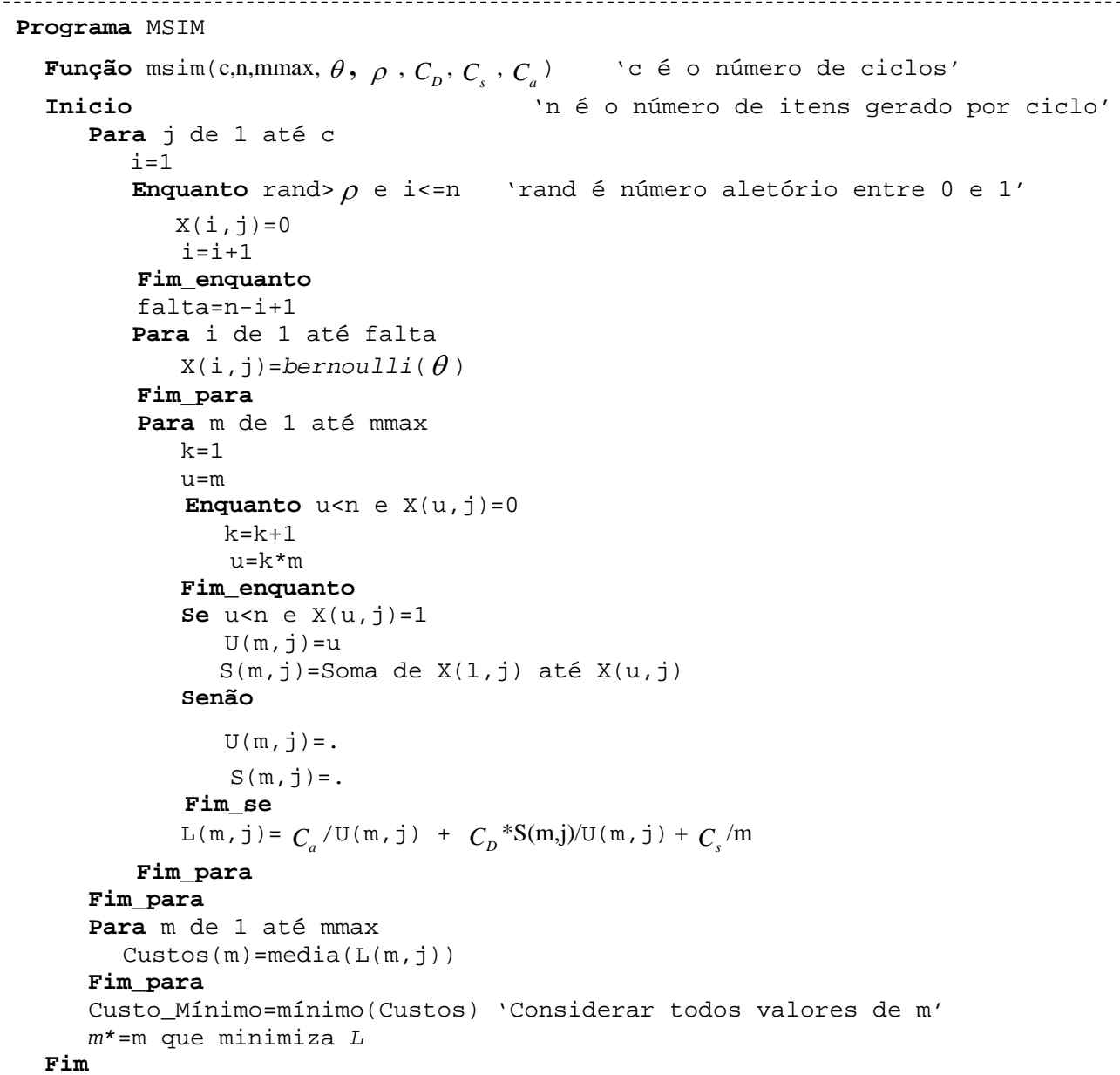

Figura 5.4: Programa em pseudocódigo para obter $0 \mathrm{~m}^{*}$ através de simulação estocástica.

No programa, simulamos um número $c$ de ciclos e para cada um dos ciclos, calculamos o custo por item considerando cada valor de $m$. Em seguida, obtivemos a média 
desses custos por item para cada valor de $m$. Ao final, o intervalo de inspeção ótimo foi aquele que apresentou o menor custo médio por item.

Limitamos o tamanho do vetor que será utilizado para simulação a um valor fixado passado no início do programa ( $n$ ). O valor de $n$ deve ser escolhido de acordo com os hiperparâmetros e o valor máximo de $m$ ( $\operatorname{mmax}$ ) que se deseja avaliar, para garantir que ao percorrer o vetor em intervalos de $m$ observações seja possível encontrar itens não conformes em inspeções para todos os valores de $m$, determinando o momento em que cada ciclo é finalizado e permitindo calcular o custo esperado por item para todos os valores de $m$ nesse intervalo.

O programa foi implementado no software Matlab R12, que já tem implementado a função betarnd que gera números aleatórios com distribuição Beta(a,b), a função rand que gera números aleatórios com distribuição $\operatorname{Uniforme}(0,1)$ e a função $\operatorname{binornd}(n, \theta)$, com $n=1$, que gera números aleatórios segundo uma distribuição de $\operatorname{Bernoulli}(\theta)$. O código fonte encontra-se no Apêndice E.

\subsection{Exemplos Numéricos}

Nessa seção, avaliaremos o desempenho de cada método e da simulação estocástica através de exemplos numéricos.

O exemplo utilizado será o mesmo apresentado por Nayebpour e Woodall (1993, pp. 59). Porém, como estamos considerando modelos em que não há atraso e inspeção retrospectiva, essas informações não serão apresentadas no nosso exemplo.

Exemplo 1: Em um processo de produção envolvendo a inspeção de soldagens em componentes de placas de circuito impresso, sabe-se que o custo de inspeção por placa (Cs ) é de R\$ 30,00, a perda causada pela produção de uma placa não conforme ( $C_{d}$ ) é de $\mathrm{R} \$ 120,00$ e o custo de ajuste $\left(C_{a}\right)$ é $\mathrm{R} \$ 500,00$.

Consideramos um intervalo de inspeção inicial $\left(m_{c}\right)$ de 50 unidades, $\theta=0,1$ e $\rho=0,05$, e utilizamos a informação do primeiro ciclo da simulação estocástica para obter um valor inicial para $\eta, \tau$ e $\sum_{i=1}^{m_{c} \tau} X_{i}$. Nessa ciclo, observamos que o processo saiu de controle na produção do $59^{\circ}$ item. Foram necessárias onze inspeções para encontrar um 
item não conforme e até a décima primeira inspeção foram produzidos 550 itens, sendo que 53 deles eram não conformes.

A recomendação de Nayebpour e Woodall é que seja obtida uma estimativa de $\theta$ utilizando dados históricos de ciclos, onde foi realizado inspeção retrospectiva total. Por isso, no modelo de Nayebpour e Woodall, utilizaremos para $\theta$ o percentual de itens não conforme encontrados após o processo sair de controle na simulação de um ciclo, ou seja, $\theta_{N W}=\frac{53}{550-59+1}=0,108$.

Para o método bayesino, consideraremos distribuições a priori uniforme ( $a_{1}=b_{1}=a_{2}=b_{2}=1$ ), e calcularemos a distribuição a posteriori com o valor de $\tau$ observado no primeiro ciclo da simulação estocástica $(\tau=11)$.

Todos os métodos utilizaram como base os dados obtidos no primeiro ciclo da simulação estocástica, em que foram simulados 150 ciclos. No método bayesiano, para a integração numérica, foram gerados 2.000 pontos.

Com base nas informações descritas nesse exemplo, obtivemos o gráfico do custo esperado por item em função de $m$ para cada um dos métodos.

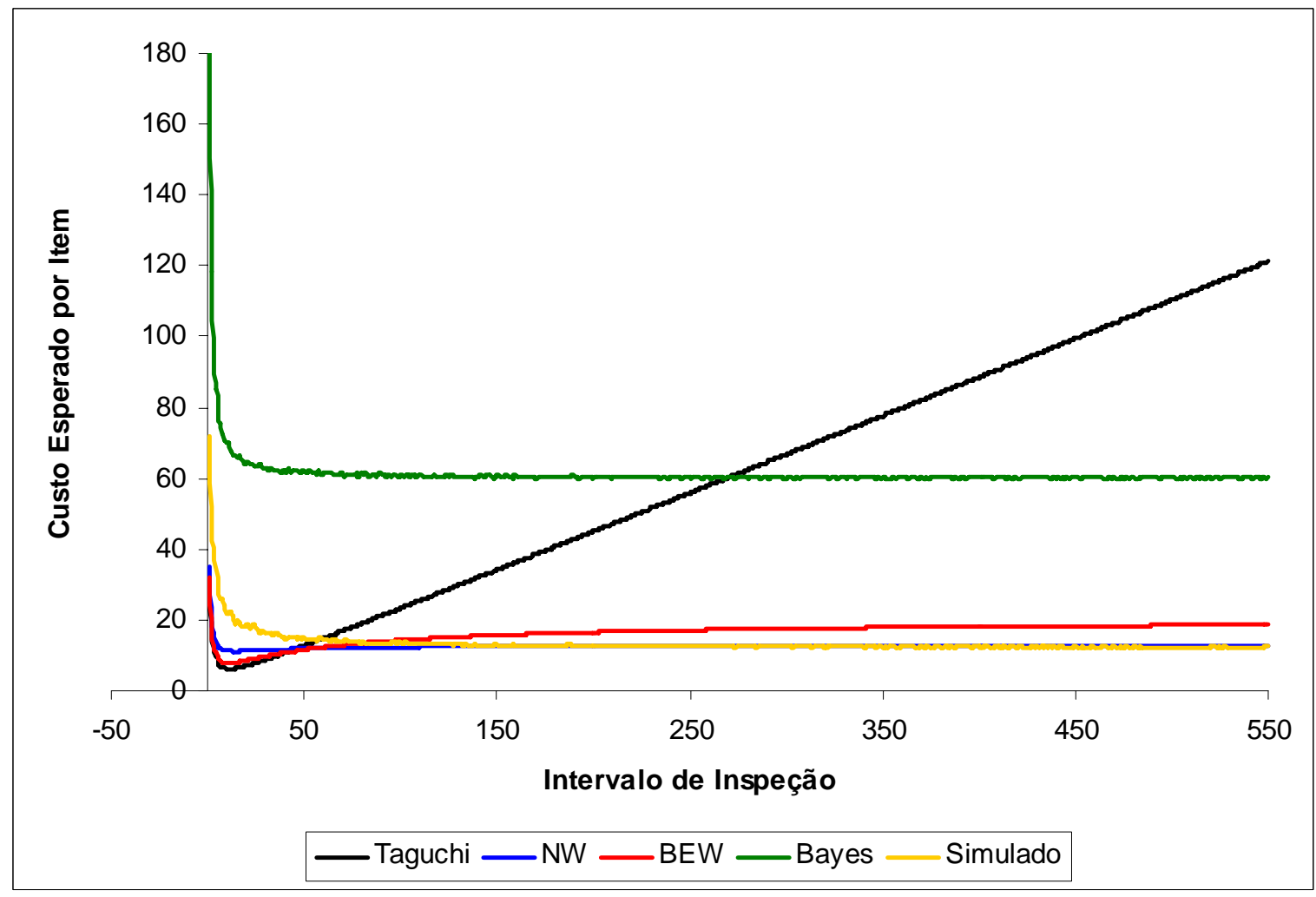

Figura 5.5: Gráfico do custo esperado por item em função de $m$ para cada um dos métodos. 
Observamos no gráfico (figura 5.5) que a curva do método bayesiano apresenta custos distantes ao da simulação estocástica, porém os comportamentos das curvas são semelhantes.

Abaixo, temos a tabela com o intervalo de inspeção ótimo e o custo esperado por item para o segundo ciclo de cada métodos estudado, e os valores de referência obtidos por simulação estocástica:

\begin{tabular}{ccc}
\hline Método & $\boldsymbol{m}^{*}$ & Custo Esperado por Item \\
\hline Taguchi & 12 & 6,25 \\
\hline NW & 14 & 11,15 \\
\hline BEW & 11 & 7,87 \\
\hline Simulado & 545 & 12,17 \\
\hline Bayesiano & 542 & 59,78 \\
\hline
\end{tabular}

Tabela 5.1: Comparação entre os métodos.

A partir da tabela 5.1, notamos que o método bayesiano apresenta intervalo de inspeção ótimo próximo ao obtido por simulação estocástica, porém, custos esperados muito maiores. A diferença em relação aos custos ocorre, provavelmente, por utilizar uma distribuição à priori não informativa e fazer apenas uma atualização da posteriori. Nos testes realizados utilizando priori informativas (distribuições Beta independentes) com os valores dos hiperparâmetros escolhidos para obter valores esperados para $\theta$ e $\rho$ próximo aos valores escolhidos no método de simulação, o custo esperado por item é próximo ao obtido por simulação estocástica.

Exemplo 2: Neste exemplo, utilizaremos as mesmas informações do exemplo 1, alterando os valores de $\theta$ para verificar o comportamento de cada um dos métodos com a alteração desse parâmetro. Os valores utilizados para $\theta$ foram $0,1,0,2,0,3,0,4$ e 0,5 com $\rho$ fixado em 0,05 .

Utilizando um intervalo de inspeção inicial $\left(m_{c}\right)$ de 10 unidades, realizamos a simulação estocástica do primeiro ciclo e obtivemos os seguintes resultados para cada valor de $\theta$ : o número de itens produzidos até o processo sair de controle $(\eta)$, o número de inspeção até encontrar o primeiro item não conforme $(\tau)$, o número de itens não conformes produzidos $\left(\sum_{i=1}^{m_{c} \tau} X_{i}\right)$, o número total de itens produzidos e o percentual de itens 
não conforme encontrados após o processo sair de controle $\left(\theta_{N W}\right)$. Na tabela abaixo, apresentamos esses resultados:

\begin{tabular}{cccccc}
\hline$\theta$ & $\eta$ & $\tau$ & $\sum_{i=1}^{m_{c} \tau} X_{i}$ & $u=m_{c} \tau$ & $\theta_{N W}$ \\
\hline 0,1 & 56 & 6 & 2 & 60 & 0,400 \\
\hline 0,2 & 6 & 2 & 4 & 20 & 0,267 \\
\hline 0,3 & 78 & 8 & 2 & 80 & 0,667 \\
\hline 0,4 & 17 & 4 & 13 & 40 & 0,542 \\
\hline 0,5 & 16 & 2 & 4 & 20 & 0,800 \\
\hline
\end{tabular}

Tabela 5.2: Valores obtidos na simulação de um ciclo para cada valor de $\theta, \operatorname{com} \rho=0,05$.

A simulação estocástica considerou 150 ciclos e no método bayesiano para a integração numérica foram gerados 2.000 pontos.

$\mathrm{Na}$ figura 5.6, temos o gráfico do intervalo de inspeção ótimo em função da probabilidade de produzir itens não conformes após o processo sair de controle $(\theta)$, para cada um dos métodos.

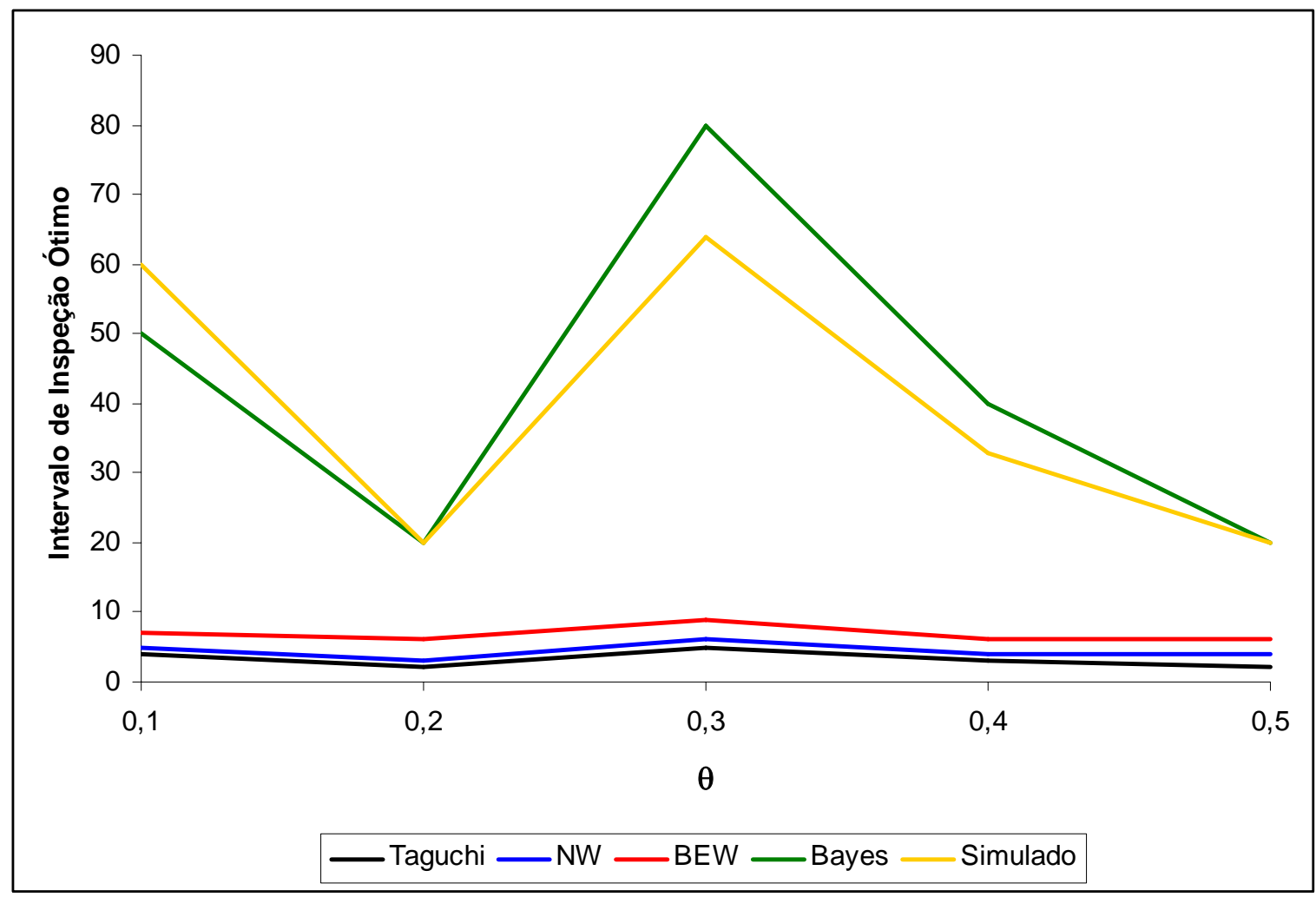

Figura 5.6: Gráfico do intervalo de inspeção ótimo em função de $\theta$.

Observamos no gráfico (figura 5.6) que os intervalos de inspeção ótimos no método bayesiano são próximos aos obtidos por simulação estocástica. 
Na figura 5.7, temos o gráfico do custo esperado por item em função da probabilidade de produzir itens não conformes após o processo sair de controle $(\theta)$, para cada um dos métodos.

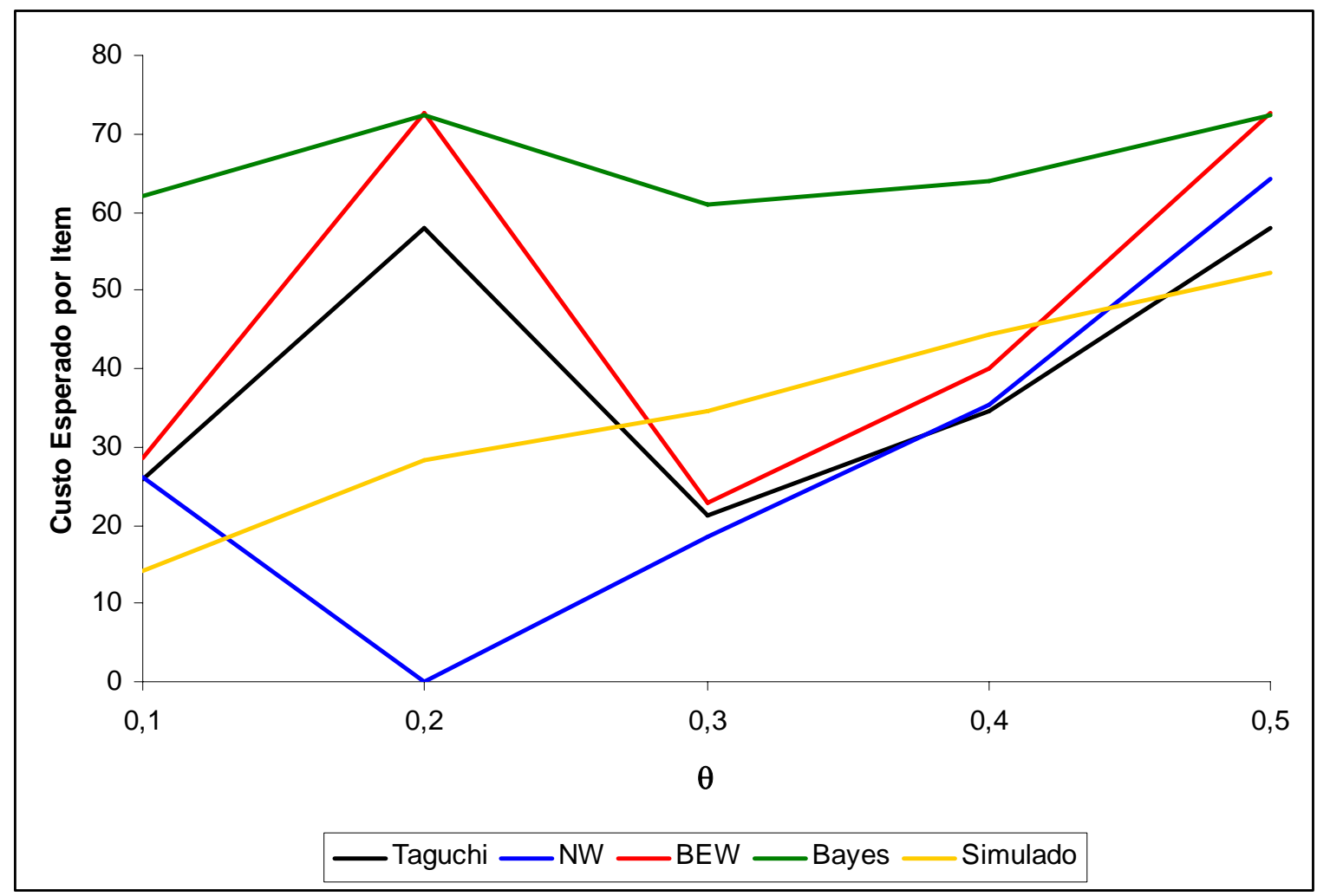

Figura 5.7: Gráfico do custo esperado por item em função de $\theta$.

Verificamos que os custos obtidos por simulação estocástica apresentam grandes diferenças em relação ao custo dos demais métodos.

No método de Nayebpour e Woodall (1993) para $\theta=0,2$ obtivemos um valor negativo para o parâmetro $\rho$, utilizando o estimador em (3.15). Com isso, tivemos um custo negativo (-387), para não distorcer o gráfico esse custo foi substituído por zero.

Provavelmente, a diferença dos custos dos quatro métodos em relação à simulação estocástica ocorre devido aos resultados estarem baseados em um único ciclo.

Escolhemos utilizar apenas um ciclo devido à complexidade da distribuição a posteriori que deveria ser atualizada a cada novo ciclo, o que dificulta a implementação do método bayesiano. 
Exemplo 3: Nos exemplos anteriores, consideramos valores $\theta>\rho$. Porém, podemos ter processos produtivos em que $\theta<\rho$ e, nesses casos, os métodos de Taguchi, Nayebpour e Woodall e o método utilizando estimativas de máxima verossimilhança (BEW) não são adequados, pois as estimativas dos parâmetros satisfazem $\theta \geq \rho$.

Para verificar o desempenho do método bayesiano nesse exemplo, utilizamos os mesmos custos do exemplo 2.

Considerando um intervalo de inspeção inicial $\left(m_{c}\right)$ de 50 unidades e $\theta=0,1$ e $\rho=0,2$, e utilizando os resultados do primeiro ciclo da simulação estocástica, observamos que o processo saiu de controle na produção do $14^{\circ}$ item. Foram necessárias cinco inspeções para encontrar um item não conforme e até a quinta inspeção foram produzidos 250 itens, sendo que 26 deles eram não conformes.

Assim como no exemplo anterior, a simulação estocástica foi realizada com 150 ciclos e no método bayesiano para a integração numérica foram gerados 2.000 pontos.

Com base nessas informações, obtivemos o gráfico do custo esperado por item em função de $m$ para o método bayesiano e para a simulação estocástica.

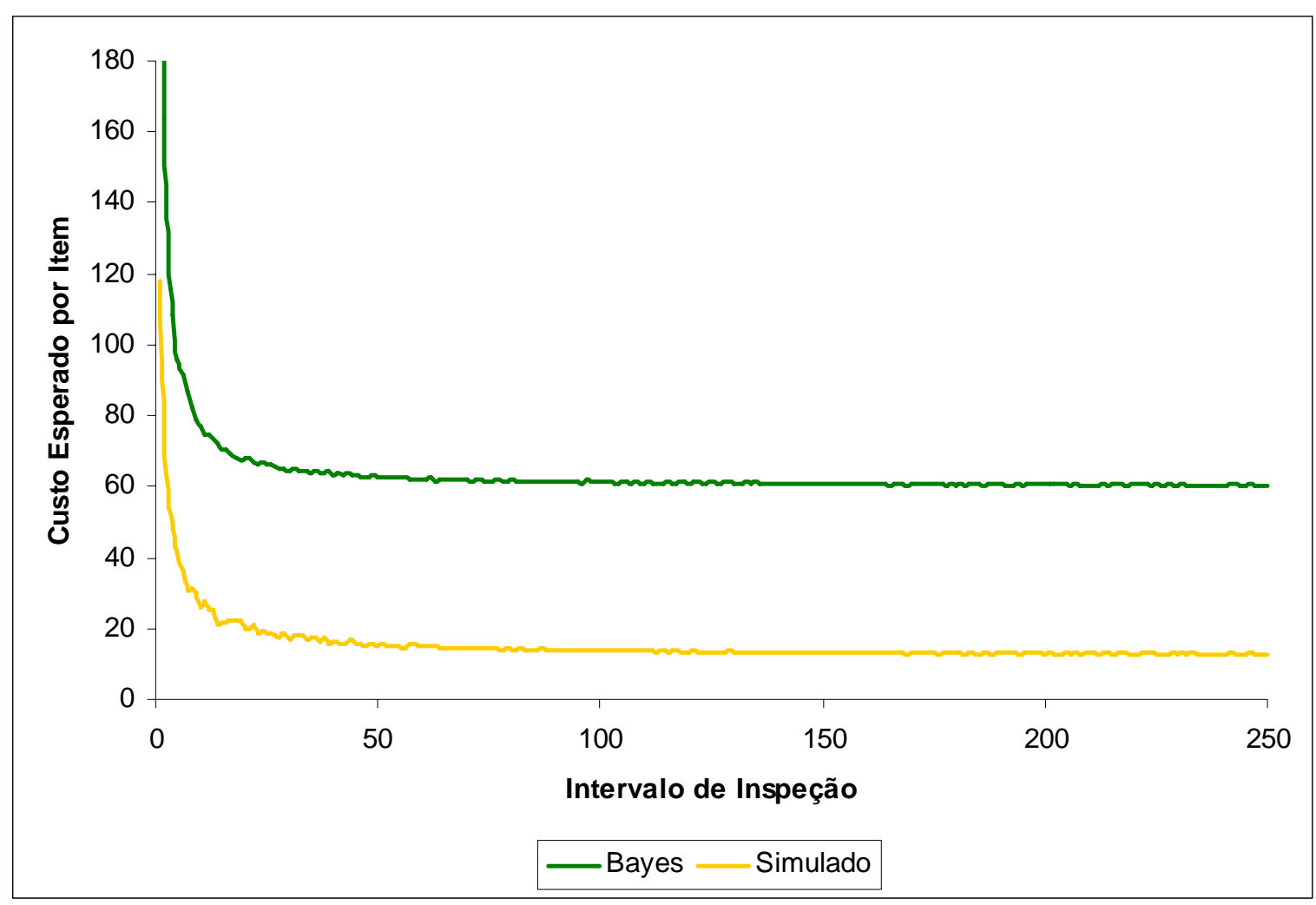

Figura 5.8: Gráfico do custo esperado por item em função de $m$. 
Observamos no gráfico (figura 5.8) que novamente há uma grande diferença entre os custos, porém, os comportamentos das curvas são semelhantes.

Na tabela 5.3, temos o intervalo de inspeção ótimo e o custo esperado por item para o método bayesiano e para a simulação estocástica.

\begin{tabular}{ccc}
\hline Método & $\boldsymbol{m}^{*}$ & Custo Esperado por Item \\
\hline Simulação & 245 & 12,63 \\
\hline Bayesiano & 249 & 60,11 \\
\hline
\end{tabular}

Tabela 5.3: Comparação entre os métodos.

A partir da tabela 5.3, notamos que, os intervalos de inspeção ótimos são próximos.

Concluímos com os exemplos, que o método bayesiano apresenta maior flexibilidade que os demais métodos, não possuindo restrição na escolha dos hiperparâmetros da distribuição a priori e permitindo considerar o caso $\theta<\rho$. Além disso, os intervalos de inspeções ótimos são próximos ao obtidos por simulação estocástica. 


\section{Capítulo 6}

\section{Conclusão}

Nesse trabalho, estudamos o método econômico desenvolvido por Taguchi para monitorar variáveis dicotômicas de atributo em tempo real (on line), determinando o intervalo ótimo de inspeção que minimiza o custo por item, em um ciclo de produção.

Apresentamos o modelo alternativo proposto por Nayebpour e Woodall (1993) para obter o custo esperado por item produzido, assumindo que o tempo necessário até o processo sair do controle tem distribuição geométrica, e derivamos os estimadores de máxima verossimilhança e de Bayes para esse modelo, desenvolvido por Borges, Esteves e Wechsler (2005).

Borges, Esteves e Wechsler (2005) encontraram também distribuição a posteriori para o parâmetro $(\theta, \rho)$, assumindo distribuições Beta a priori, conforme demonstrado no trabalho.

A maior contribuição desse trabalho foi abordar o problema de determinação do intervalo de inspeção ótimo sob a perspectiva da Teoria de Decisão Bayesiana. Foi implementado um programa utilizando integração numérica para resolver o problema. Esse programa foi aplicado a alguns exemplos.

A abordagem da Teoria de Decisão Bayesiana é, nesse caso, mais flexível, não tendo limitações como a do método de Nayebpour e Woodall (1993) e dos estimadores de máxima verossimilhança obtidos Borges, Esteves e Wechsler (2005), para os quais não é possível considerar o caso em que $\theta<\rho$. Sob a abordagem Bayesiana, não há restrições em utilizar prioris para $(\theta, \rho)$ que imponham essa condição.

Para estudos futuros, sugere-se: 
- Implementar a abordagem sob a perspectiva da Teoria de Decisão Bayesiana considerando atualizações da distribuição a posteriori baseadas em um maior número de ciclos e outras distribuições a priori. Com isso, podemos avaliar melhor desempenho dessa abordagem;

- Trabalhar com outras distribuições de probabilidade para o tempo necessário até o processo sair do controle, assumindo que a probabilidade do processo sair de controle pode mudar com o tempo. Com isso, obteríamos outra função de verossimilhança para implementar o modelo sob a perspectiva da Teoria de Decisão Bayesiana. 


\section{Apêndice A}

\section{Calculo da Esperança da Expressão (4.10)}

A expressão do risco $\pi(P, m)$, apresentada no capítulo 4, envolve a esperança de $L$ dados $\theta$ e $\rho$. Por isso, temos a necessidade de resolver a expressão abaixo:

$$
E(L \mid \theta, \rho)=\frac{C_{a}}{m} E\left(\frac{1}{\tau_{m}} \mid \theta, \rho\right)+\frac{C_{s}}{m}+\frac{C_{d}}{m} E\left(\frac{\sum_{i=1}^{m \tau_{m}} X_{i}}{\tau_{m}} \mid \theta, \rho\right)
$$

Na expressão (A.1) $\tau_{m}=\inf \left\{k \in \mathrm{N}: X_{k m}=1\right\}$.

Determinando a esperança da primeira parcela, temos:

$$
\begin{aligned}
& E\left(\frac{1}{\tau_{m}} \mid \theta, \rho\right)=\sum_{k=1}^{\infty} \frac{1}{k} P\left(\tau_{m}=k \mid \theta, \rho\right) \\
= & \sum_{k=1}^{\infty} \frac{\theta}{k}\left(1-(1-\rho)^{m}\right) \sum_{j=0}^{k-1}(1-\rho)^{m j}(1-\theta)^{k-1-j}
\end{aligned}
$$

Desenvolvendo a somatória interna para $(1-\rho)^{m} \neq(1-\theta)$, obtemos:

$$
\begin{array}{r}
\sum_{j=0}^{k-1}(1-\rho)^{m j}(1-\theta)^{k-1-j}=(1-\theta)^{k-1}\left(\frac{(1-\rho)^{m k}}{(1-\theta)^{k}}-1\right)\left(\frac{(1-\rho)^{m}}{1-\theta}-1\right)^{-1} \\
=(1-\theta)^{k-1}\left(\frac{(1-\rho)^{m k}-(1-\theta)^{k}}{(1-\theta)^{k}}\right)\left(\frac{1-\theta}{(1-\rho)^{m}-(1-\theta)}\right)=\frac{(1-\rho)^{m k}-(1-\theta)^{k}}{(1-\rho)^{m}-(1-\theta)}
\end{array}
$$

Então: 


$$
\begin{array}{r}
E\left(\frac{1}{\tau_{m}} \mid \theta, \rho\right)=\sum_{k=1}^{\infty} \frac{\theta}{k}\left(1-(1-\rho)^{m}\right)\left(\frac{(1-\rho)^{m k}-(1-\theta)^{k}}{(1-\rho)^{m}-(1-\theta)}\right) \\
=\frac{\theta\left(1-(1-\rho)^{m}\right)}{(1-\rho)^{m}-(1-\theta)}\left(\sum_{k=1}^{\infty} \frac{(1-\rho)^{m k}}{k}-\sum_{k=1}^{\infty} \frac{(1-\theta)^{k}}{k}\right)
\end{array}
$$

A soma em séries de potência para o logaritmo natural de $1+y$ é $\ln (1+y)=\sum_{i=0}^{\infty} \frac{(-1)^{i} y^{i+1}}{i+1}$. Utilizando esse resultado podemos resolver as duas somatórias da expressão (A.2):

$$
\begin{gathered}
\sum_{k=1}^{\infty} \frac{(1-\rho)^{m k}}{k}=\sum_{k=0}^{\infty} \frac{(1-\rho)^{m(k+1)}}{k+1} \\
=\sum_{k=0}^{\infty} \frac{(-1)^{k}(-1)^{k}\left[(1-\rho)^{m}\right]^{k+1}}{k+1} \\
=-\sum_{k=0}^{\infty} \frac{(-1)^{k}(-1)^{k+1}\left[(1-\rho)^{m}\right]^{k+1}}{k+1}=-\ln \left(1-(1-\rho)^{m}\right)
\end{gathered}
$$

Através de raciocínio análogo concluímos que:

$$
\sum_{k=1}^{\infty} \frac{(1-\theta)^{k}}{k}=-\ln (\theta)
$$

Substituindo os resultados obtidos em (A.3) e (A.4) na expressão (A.2) obtemos:

$$
E\left(\frac{1}{\tau_{m}} \mid \theta, \rho\right)=\frac{\theta\left(1-(1-\rho)^{m}\right)}{(1-\rho)^{m}-(1-\theta)} \ln \left(\frac{\theta}{1-(1-\rho)^{m}}\right)
$$

Calculamos a esperança da terceira parcela da equação (A.1) via condicionamento. Lembrando que $\eta=\inf \left\{n \in \mathrm{N}: P\left(X_{n}=1 \mid \theta, \rho\right)=\theta>0\right\}$, temos: 


$$
\begin{aligned}
& E\left(\frac{\sum_{i=1}^{m \tau_{m}} X_{i}}{\tau_{m}} \mid \theta, \rho\right)=E\left(E\left(\frac{\sum_{i=1}^{m \tau_{m}} X_{i}}{\tau_{m}} \mid \theta, \rho, \tau, \eta\right)\right) \\
& =E\left(E\left(\frac{\sum_{i=1}^{\eta-1} X_{i}+\sum_{i=\eta}^{m \tau_{m}} X_{i}}{\tau_{m}} \mid \theta, \rho, \tau, \eta\right)\right)
\end{aligned}
$$

A última esperança da expressão acima é calculada através da distribuição conjunta de $\left(\tau_{m}, \eta\right) \mid(\theta, \rho)$.

Dado $\eta$, sabemos que até a produção do $\eta$-ésimo item, ou seja, até o processo sair de controle, são produzidas apenas itens conformes, então $\sum_{i=1}^{\eta-1} X_{i}=0$. Ao produzir o $\eta$-ésimo item, o processo sai de controle e passa a produzir itens não conformes com probabilidade $\theta$. Portanto, para $i \geq \eta, i \neq j m, j \in \mathrm{N}, X_{i} \mid \theta, \rho, \tau_{m}, \eta \sim \operatorname{Bernoulli}(\theta)$. Entretanto, nos momentos em que foram feitas as inspeções, $i=j m, j \in \mathrm{N}, X_{i}=0$, exceto na última inspeção em que é detectado um item não conforme, ou seja, $X_{m \tau_{m}}=1$. Além disso, após o processo sair de controle tivemos $\tau_{m}-\left\lfloor\frac{\eta-1}{m}\right\rfloor$ inspeções. Voltando à expressão (A.6), temos:

$$
\begin{aligned}
E\left(E\left(\frac{\sum_{i=1}^{\eta-1} X_{i}+\sum_{i=\eta}^{m \tau_{m}} X_{i}}{\tau_{m}} \mid \theta, \rho, \tau, \eta\right)\right) \\
=E\left(\frac{1}{\tau_{m}}\left(0(\eta-1)+1+\theta\left(m k-\eta+1-\tau_{m}+\left\lfloor\frac{\eta-1}{m}\right\rfloor\right)\right) \mid \theta, \rho, \tau_{m}, \eta\right)
\end{aligned}
$$


Com esse resultado, temos:

$$
\begin{gathered}
\left.E\left(\frac{\sum_{i=1}^{m \tau_{m}} X_{i}}{\tau_{m}} \mid \theta, \rho\right)=E\left(\left(\frac{1}{\tau_{m}}+m \theta+\frac{\theta-\eta \theta}{\tau_{m}}-\theta+\frac{\theta}{\tau_{m}} \mid \frac{\eta-1}{m}\right\rfloor\right) \mid \theta, \rho\right) \\
=E\left(\frac{1}{\tau_{m}} \mid \theta, \rho\right)+m \theta+\theta E\left(\frac{1}{\tau_{m}} \mid \theta, \rho\right)-\theta E\left(\frac{\eta}{\tau_{m}} \mid \theta, \rho\right)-\theta+\theta E\left(\frac{1}{\tau_{m}}\left|\frac{\eta-1}{m}\right| \mid \theta, \rho\right) \\
=(m-1) \theta+(1+\theta) E\left(\frac{1}{\tau_{m}} \mid \theta, \rho\right)+\theta E\left(\frac{\left\lfloor\frac{\eta-1}{m}\right\rfloor-\eta}{\tau_{m}} \mid \theta, \rho\right)
\end{gathered}
$$

Se $(i-1) m+1 \leq \eta-1 \leq i m$, então $\frac{(i-1) m+1-1}{m} \leq \frac{\eta-1}{m} \leq \frac{i m-1}{m}$, e $\left\lfloor\frac{\eta-1}{m}\right\rfloor=i-1$.

Utilizaremos esse resultado para resolver a esperança da terceira parcela da expressão (A.8):

$$
E\left(\frac{\left\lfloor\frac{\eta-1}{m}\right\rfloor-\eta}{\tau_{m}} \mid \theta, \rho\right)=E\left(E\left(\frac{\left\lfloor\frac{\eta-1}{m}\right\rfloor-\eta}{\tau_{m}} \mid \theta, \rho, \tau_{m}\right)\right)
$$

Dado $\tau_{m}=k$, temos:

$$
\begin{gathered}
E\left(\frac{\left\lfloor\frac{\eta-1}{m}\right\rfloor-\eta}{\tau_{m}} \mid \theta, \rho, \tau_{m}=k\right)=\frac{1}{k}\left(E\left(\left\lfloor\frac{\eta-1}{m}\right\rfloor-\eta\right) \mid \theta, \rho, \tau_{m}=k\right) \\
=\frac{1}{k} \sum_{i=1}^{k} \sum_{n=(i-1) m+1}^{i m}(i-1-n) P\left(\eta=n \mid \theta, \rho, \tau_{m}=k\right)
\end{gathered}
$$

Mas, 


$$
\begin{array}{r}
P\left(\eta=n \mid \theta, \rho, \tau_{m}=k\right)=\frac{P\left(\eta=n, \tau_{m}=k \mid \theta, \rho\right)}{P\left(\tau_{m}=k \mid \theta, \rho\right)} \\
=\frac{P(\eta=n \mid \theta, \rho) P\left(\tau_{m}=k \mid \theta, \rho, \eta=n\right)}{P\left(\tau_{m}=k \mid \theta, \rho\right)}
\end{array}
$$

Para $\quad k<n, \quad P\left(\tau_{m}=k \mid \theta, \rho, \eta=n\right)=0 \quad$ e, $\quad$ para $\quad k \geq n$,

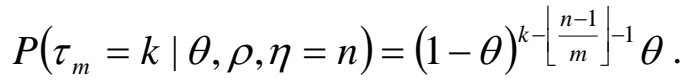

Como $\left\lfloor\frac{\eta-1}{m}\right\rfloor=i-1$, se $(i-1) m+1 \leq n \leq i m$, então para $k \geq n$ :

$$
P\left(\eta=n \mid \theta, \rho, \tau_{m}=k\right)=\frac{(1-\rho)^{n-1} \rho(1-\theta)^{k-i} \theta}{P\left(\tau_{m}=k \mid \theta, \rho\right)}
$$

Voltando a expressão (A.9), temos:

$$
\begin{aligned}
& E\left(\frac{\left\lfloor\frac{\eta-1}{m}\right\rfloor-\eta}{\tau_{m}} \mid \theta, \rho, \tau_{m}=k\right)=\frac{1}{k} \sum_{i=1}^{k} \sum_{n=(i-1) m+1}^{i m} \frac{(i-1-n)(1-\rho)^{n-1} \rho(1-\theta)^{k-i} \theta}{P\left(\tau_{m}=k \mid \theta, \rho\right)} \\
&= \frac{\theta \rho}{k P\left(\tau_{m}=k \mid \theta, \rho\right)} \sum_{i=1}^{k}(1-\theta)^{k-i}\left(\sum_{n=(i-1) m+1}^{i m}(i-1)(1-\rho)^{n-1}-\sum_{n=(i-1) m+1}^{i m} n(1-\rho)^{n-1}\right) \\
&= \frac{\theta \rho}{k P\left(\tau_{m}=k \mid \theta, \rho\right)} \sum_{i=1}^{k}(1-\theta)^{k-i}\left((i-1) \sum_{n=0}^{m-1}(1-\rho)^{n+(i-1) m}-\sum_{n=(i-1) m+1}^{i m} n(1-\rho)^{n-1}\right) \\
&=\frac{\theta \rho}{k P\left(\tau_{m}=k \mid \theta, \rho\right)} \sum_{i=1}^{k}(1-\theta)^{k-i}\left(\frac{(i-1)(1-\rho)^{(i-1) m}\left(1-(1-\rho)^{m}\right)}{1-(1-\rho)}-\sum_{n=(i-1) m+1}^{i m} n(1-\rho)^{n-1}\right)
\end{aligned}
$$

Mas,

$$
\begin{gathered}
\sum_{n=(i-1) m+1}^{i m} n(1-\rho)^{n-1}=-\sum_{n=(i-1) m+1}^{i m} \frac{\partial}{\partial \rho}(1-\rho)^{n} \\
=-\frac{\partial}{\partial \rho} \sum_{n=(i-1) m+1}^{i m}(1-\rho)^{n}=-\frac{\partial}{\partial \rho} \sum_{n=0}^{m-1}(1-\rho)^{n+(i-1) m+1}
\end{gathered}
$$




$$
\begin{gathered}
=-\frac{\partial}{\partial \rho}\left(\frac{(1-\rho)^{(i-1) m+1}\left(1-(1-\rho)^{m}\right)}{1-(1-\rho)}\right) \\
=-\frac{\partial}{\partial \rho}\left(\frac{(1-\rho)^{(i-1) m+1}-(1-\rho)^{i m+1}}{\rho}\right) \\
=-\frac{\rho\left(-((i-1) m+1)(1-\rho)^{(i-1) m}+(i m+1)(1-\rho)^{i m}\right)-(1-\rho)^{(i-1) m+1}+(1-\rho)^{i m+1}}{\rho^{2}} \\
=-\frac{(m i+1)(1-\rho)^{m(i-1)}\left((1-\rho)^{m}-1\right)+m(1-\rho)^{m(i-1)}}{\rho}+\frac{(1-\rho)^{m(i-1)+1}\left(1-(1-\rho)^{m}\right)}{\rho^{2}}
\end{gathered}
$$

\section{Então:}

$$
\begin{gathered}
E\left(\frac{\left.\mid \frac{\eta-1}{m}\right\rfloor-\eta}{\tau_{m}} \mid \theta, \rho, \tau_{m}=k\right) \\
=\frac{\theta \rho}{k P\left(\tau_{m}=k \mid \theta, \rho\right)} \sum_{i=1}^{k}(1-\theta)^{k-i}\left(\frac{(i-1)(1-\rho)^{m(i-1)}\left(1-(1-\rho)^{m}\right)}{\rho}+\right. \\
\left.+\frac{(m i+1)(1-\rho)^{m(i-1)}\left((1-\rho)^{m}-1\right)+m(1-\rho)^{m(i-1)}}{\rho}-\frac{(1-\rho)^{m(i-1)+1}\left(1-(1-\rho)^{m}\right)}{\rho^{2}}\right) \\
=\frac{\theta \rho(1-\theta)^{k}}{k P\left(\tau_{m}=k \mid \theta, \rho\right)}\left(\frac{1-(1-\rho)^{m}}{\rho(1-\theta)} \sum_{i=1}^{k} \frac{(i-1)(1-\rho)^{m(i-1)}-\frac{1-(1-\rho)^{m}}{\rho(1-\theta)} \sum_{i=1}^{k} \frac{(m i+1)(1-\rho)^{m(i-1)}}{(1-\theta)^{i-1}}+}{\left.+\frac{m}{\rho(1-\theta)} \sum_{i=1}^{k} \frac{(1-\rho)^{m(i-1)}}{(1-\theta)^{i-1}}-\frac{\left(1-(1-\rho)^{m}\right)(1-\rho)}{\rho^{2}(1-\theta)} \sum_{i=1}^{k} \frac{(1-\rho)^{m(i-1)}}{(1-\theta)^{i-1}}\right)}\right. \\
=\frac{\theta \rho(1-\theta)^{k}}{k P\left(\tau_{m}=k \mid \theta, \rho\right)}\left(\frac{1-(1-\rho)^{m}}{\rho(1-\theta)}\left(\sum_{i=1}^{k} \frac{(i-m i)\left((1-\rho)^{m}\right)^{i-1}}{(1-\theta)^{i-1}}-2 \sum_{i=1}^{k} \frac{\left.(1-\rho)^{m}\right)^{i-1}}{(1-\theta)^{i-1}}\right)+\right. \\
\left.+\frac{m}{\rho(1-\theta)}\left(1-\frac{(1-\rho)^{m k}}{(1-\theta)^{k}}\right)\left(1-\frac{(1-\rho)^{m}}{1-\theta}\right)^{-1}-\frac{\left(1-(1-\rho)^{m}\right)(1-\rho)}{\rho^{2}(1-\theta)}\left(1-\frac{(1-\rho)^{m k}}{(1-\theta)^{k}}\right)\left(1-\frac{(1-\rho)^{m}}{1-\theta}\right)^{-1}\right)
\end{gathered}
$$




$$
\begin{gathered}
=\frac{\theta \rho(1-\theta)^{k}}{k P\left(\tau_{m}=k \mid \theta, \rho\right)}\left(\frac{1-(1-\rho)^{m}}{\rho(1-\theta)}\left(\sum_{i=1}^{k} \frac{(i-m i)\left((1-\rho)^{m}\right)^{i-1}}{(1-\theta)^{i-1}}-2\left(1-\frac{(1-\rho)^{m k}}{(1-\theta)^{k}}\right)\left(1-\frac{(1-\rho)^{m}}{1-\theta}\right)^{-1}\right)+\right. \\
\left.+\frac{m\left((1-\theta)^{k}-(1-\rho)^{m k}\right)}{\rho(1-\theta)^{k}\left((1-\theta)-(1-\rho)^{m}\right)}-\frac{\left(1-(1-\rho)^{m}\right)(1-\rho)\left((1-\theta)^{k}-(1-\rho)^{m k}\right)}{\rho^{2}(1-\theta)^{k}\left((1-\theta)-(1-\rho)^{m}\right)}\right) \\
=\frac{\theta \rho}{k P\left(\tau_{m}=k \mid \theta, \rho\right)} \frac{(1-\theta)^{k}-(1-\rho)^{m k}}{(1-\theta)-(1-\rho)^{m}}\left(\frac{m}{\rho}-\frac{2 \rho\left(1-(1-\rho)^{m}\right)+(1-\rho)\left(1-(1-\rho)^{m}\right)}{\rho^{2}}\right)+ \\
+\frac{\theta(1-\theta)^{k}\left(1-(1-\rho)^{m}\right)}{k(1-\theta) P(\tau=k \mid \theta, \rho)} \sum_{i=1}^{k}(i-m i)\left(\frac{(1-\rho)^{m}}{1-\theta}\right)^{i-1}
\end{gathered}
$$

Mas,

$$
\sum_{i=1}^{k}(i-m i)\left(\frac{(1-\rho)^{m}}{1-\theta}\right)^{i-1}=(1-m) \sum_{i=1}^{k} i\left(\frac{(1-\rho)^{m}}{1-\theta}\right)^{i-1}
$$

Definindo $\psi=\frac{(1-\rho)^{m}}{1-\theta}$, temos:

$$
\begin{gathered}
(1-m) \sum_{i=1}^{k} i \psi^{i-1}=(1-m) \sum_{i=1}^{k} \frac{\partial}{\partial \psi}\left(\psi^{i}\right) \\
=(1-m) \frac{\partial}{\partial \psi} \sum_{i=1}^{k} \psi^{i} \\
=(1-m) \frac{\partial}{\partial \psi}\left(\frac{\psi\left(1-\psi^{k}\right)}{1-\psi}\right) \\
=(1-m)\left(\frac{(1-\psi)\left(1-(k+1) \psi^{k}\right)+\psi\left(1-\psi^{k}\right)}{(1-\psi)^{2}}\right) \\
=(1-m) \frac{1-(k+1) \psi^{k}+k \psi^{k+1}}{(1-\psi)^{2}}
\end{gathered}
$$


Voltando novamente a expressão (A.12), temos:

$$
\begin{gathered}
E\left(\frac{\left.\mid \frac{\eta-1}{m}\right\rfloor-\eta}{\tau_{m}} \mid \theta, \rho, \tau_{m}=k\right) \\
=\frac{\theta \rho\left((1-\theta)^{k}-(1-\rho)^{m k}\right)}{k P\left(\tau_{m}=k \mid \theta, \rho\right)\left((1-\theta)-(1-\rho)^{m}\right)}\left(\frac{m}{\rho}-\frac{(1+\rho)\left(1-(1-\rho)^{m}\right)}{\rho^{2}}\right)+ \\
+\frac{\theta(1-\theta)^{k}\left(1-(1-\rho)^{m}\right)(1-m)}{k(1-\theta) P\left(\tau_{m}=k \mid \theta, \rho\right)}\left(\frac{(1-\theta)^{2}-(k+1)(1-\rho)^{m k}(1-\theta)^{-k+2}+k(1-\rho)^{m k+m}(1-\theta)^{-k+1}}{\left(1-\theta-(1-\rho)^{m}\right)^{2}}\right) \\
=\frac{\theta\left((1-\theta)^{k}-(1-\rho)^{m k}\right)}{k P\left(\tau_{m}=k \mid \theta, \rho\right)\left((1-\theta)-(1-\rho)^{m}\right)\left(\frac{m \rho-(1+\rho)\left(1-(1-\rho)^{m}\right)}{\rho}\right)+} \\
-\frac{\theta(m-1)\left(1-(1-\rho)^{m}\right)}{k P\left(\tau_{m}=k \mid \theta, \rho\right)}\left(\frac{(1-\theta)^{k+1}-(k+1)(1-\theta)(1-\rho)^{m k}+k(1-\rho)^{m(k+1)}}{\left(1-\theta-(1-\rho)^{m}\right)^{2}}\right)
\end{gathered}
$$

Com esse resultado, obtemos:

$$
\begin{aligned}
& \quad E\left(\frac{\left\lfloor\frac{\eta-1}{m}\right\rfloor-\eta}{\tau_{m}} \mid \theta, \rho\right) \\
& =\sum_{k=1}^{\infty}\left(\frac{P\left(\tau_{m}=k \mid \theta, \rho\right)}{k} \frac{\theta\left((1-\theta)^{k}-(1-\rho)^{m k}\right)}{P\left(\tau_{m}=k \mid \theta, \rho\right)\left((1-\theta)-(1-\rho)^{m}\right)}\left(\frac{m \rho-(1+\rho)\left(1-(1-\rho)^{m}\right)}{\rho}\right)+\right. \\
& \left.-\frac{\theta(m-1)\left(1-(1-\rho)^{m}\right)}{k P\left(\tau_{m}=k \mid \theta, \rho\right)}\left(\frac{(1-\theta)^{k+1}-(k+1)(1-\theta)(1-\rho)^{m k}+k(1-\rho)^{m(k+1)}}{\left(1-\theta-(1-\rho)^{m}\right)^{2}}\right)\right) \\
& =\frac{\theta}{(1-\theta)-(1-\rho)^{m}}\left(\frac{m \rho-(1+\rho)\left(1-(1-\rho)^{m}\right)}{\rho}\right) \sum_{k=1}^{\infty} \frac{(1-\theta)^{k}-(1-\rho)^{m k}}{k}+ \\
& -\frac{\theta(m-1)\left(1-(1-\rho)^{m}\right)}{\left((1-\theta)-(1-\rho)^{m}\right)^{2}} \sum_{k=1}^{\infty} \frac{(1-\theta)^{k+1}-(k+1)(1-\theta)(1-\rho)^{m k}+k(1-\rho)^{m(k+1)}}{k}
\end{aligned}
$$




$$
\begin{gathered}
=\frac{\theta}{(1-\theta)-(1-\rho)^{m}}\left(\frac{m \rho-(1+\rho)\left(1-(1-\rho)^{m}\right)}{\rho}\right)\left(\sum_{k=1}^{\infty} \frac{(1-\theta)^{k}}{k}-\sum_{k=1}^{\infty} \frac{(1-\rho)^{m k}}{k}\right)+ \\
-\frac{\theta(m-1)\left(1-(1-\rho)^{m}\right)}{\left((1-\theta)-(1-\rho)^{m}\right)^{2}}\left((1-\theta) \sum_{k=1}^{\infty} \frac{(1-\theta)^{k}}{k}-(1-\theta) \sum_{k=1}^{\infty} \frac{(1-\rho)^{m k}}{k}-(1-\theta) \sum_{k=1}^{\infty}(1-\rho)^{m k}\right. \\
\left.+(1-\rho)^{m} \sum_{k=1}^{\infty}(1-\rho)^{m k}\right) \\
=\frac{\theta}{(1-\theta)-(1-\rho)^{m}}\left(\frac{m \rho-(1+\rho)\left(1-(1-\rho)^{m}\right)}{\rho}\right)\left(-\ln (\theta)+\ln \left(1-(1-\rho)^{m}\right)\right)+ \\
-\frac{\theta(m-1)\left(1-(1-\rho)^{m}\right)}{\left((1-\theta)-(1-\rho)^{m}\right)^{2}}\left(-(1-\theta) \ln (\theta)+(1-\theta) \ln \left(1-(1-\rho)^{m}\right)+\frac{\left((1-\rho)^{m}-(1-\theta)\right)(1-\rho)^{m}}{1-(1-\rho)^{m}}\right)
\end{gathered}
$$

Então, voltando à expressão A.1:

$$
\begin{array}{r}
E(L \mid \theta, \rho)=\frac{C_{a}}{m} E\left(\frac{1}{\tau_{m}} \mid \theta, \rho\right)+\frac{C_{s}}{m}+\frac{C_{d}}{m} E\left(\frac{\sum_{i=1}^{m \tau_{m}} X_{i}}{\tau_{m}} \mid \theta, \rho\right) \\
=\frac{C_{a}}{m} \frac{\theta\left(1-(1-\rho)^{m}\right)}{(1-\rho)^{m}-(1-\theta)} \ln \left(\frac{\theta}{1-(1-\rho)^{m}}\right)+\frac{C_{s}}{m}+\frac{C_{d}}{m}(\theta(m-1)+(1+\theta) \\
\frac{\theta\left(1-(1-\rho)^{m}\right)}{(1-\rho)^{m}-(1-\theta)} \ln \left(\frac{\theta}{1-(1-\rho)^{m}}\right)+\frac{\theta^{2}\left(m \rho-(1-\rho)\left(1-(1-\rho)^{m}\right)\right)}{\rho\left((1-\rho)^{m}-(1-\theta)\right)} \ln \left(\frac{\theta}{1-(1-\rho)^{m}}\right)+ \\
\left.-\frac{\theta^{2}(m-1)\left(1-(1-\rho)^{m}\right)}{\left((1-\rho)^{m}-(1-\theta)\right)^{2}}\left(-(1-\theta) \ln \left(\frac{\theta}{1-(1-\rho)^{m}}\right)+\frac{\left((1-\rho)^{m}-(1-\theta)\right)(1-\rho)^{m}}{1-(1-\rho)^{m}}\right)\right)
\end{array}
$$

Desta forma, a expressão final para a esperança de $L \mid \theta, \rho$ é:

$$
\begin{aligned}
& E(L \mid \theta, \rho)=\frac{C_{s}}{m}+\frac{C_{a}+C_{d}}{m} \frac{\theta\left(1-(1-\rho)^{m}\right)}{(1-\rho)^{m}-(1-\theta)} \ln \left(\frac{\theta}{1-(1-\rho)^{m}}\right)+\frac{\theta(m-1) C_{d}}{m}+ \\
& -\frac{\theta C_{d}}{m}\left(\frac{\theta\left(1-m \rho-(1-\rho)^{m}\right)}{\rho\left((1-\theta)-(1-\rho)^{m}\right)} \ln \left(\frac{1-(1-\rho)^{m}}{\theta}\right)+\frac{\theta(1-\theta)(m-1)\left(1-(1-\rho)^{m}\right)}{\left((1-\theta)-(1-\rho)^{m}\right)^{2}} \ln \left(\frac{1-(1-\rho)^{m}}{\theta}\right)+\right. \\
& \left.-\frac{\theta(m-1)(1-\rho)^{m}}{(1-\theta)-(1-\rho)^{m}}\right)
\end{aligned}
$$




\section{Apêndice B}

\section{Método de Nayebpour e Woodall}

Neste apêndice, apresentamos a função desenvolvida no software MATLAB R12 para implementar o algoritmo apresentado na Figura 5.1:

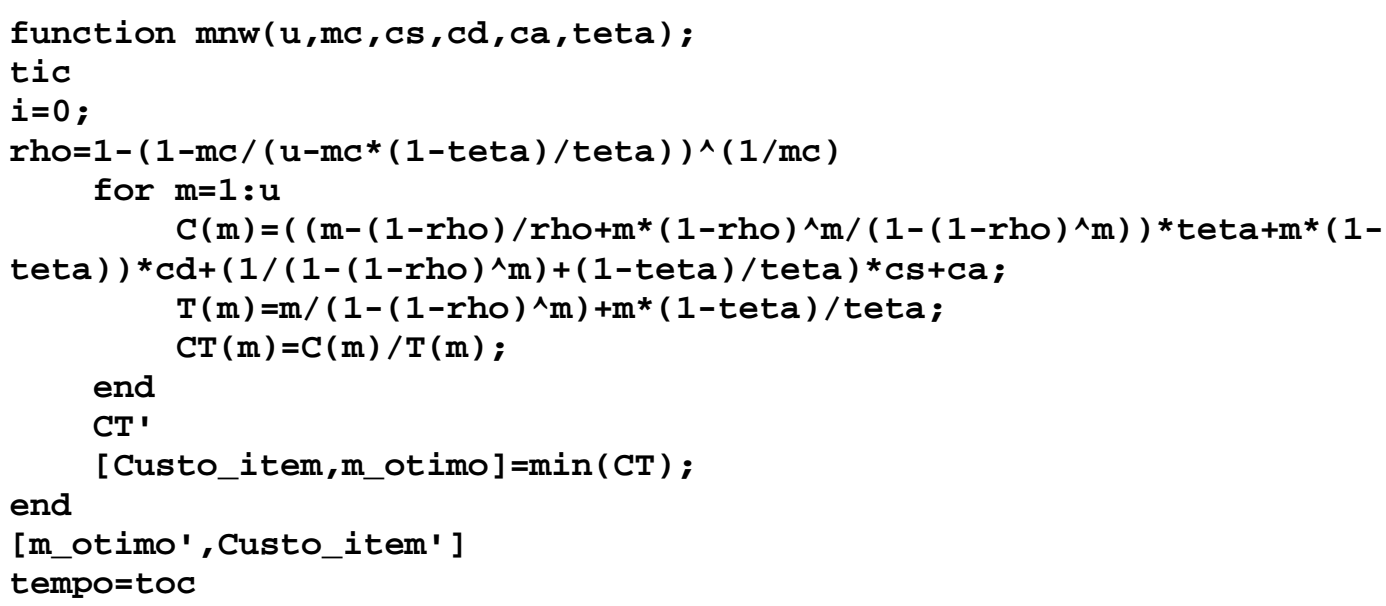




\section{Apêndice C}

\section{Método de Borges, Esteves e Wechsler}

Neste apêndice, apresentamos a função desenvolvida no software MATLAB R12 para implementar o algoritmo apresentado na Figura 5.2:

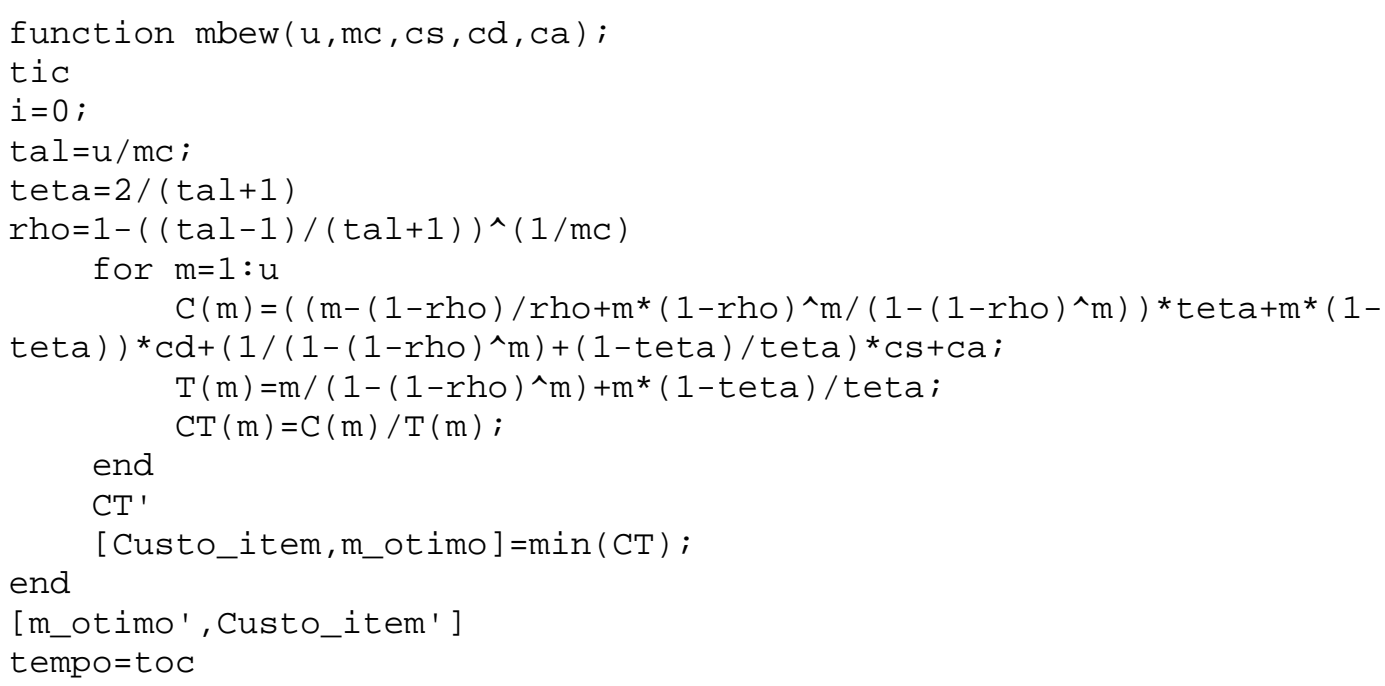




\section{Apêndice D}

\section{Abordagem Bayesiana}

Neste apêndice, apresentamos a função desenvolvida no software MATLAB R12 para implementar o algoritmo apresentado na Figura 5.3:

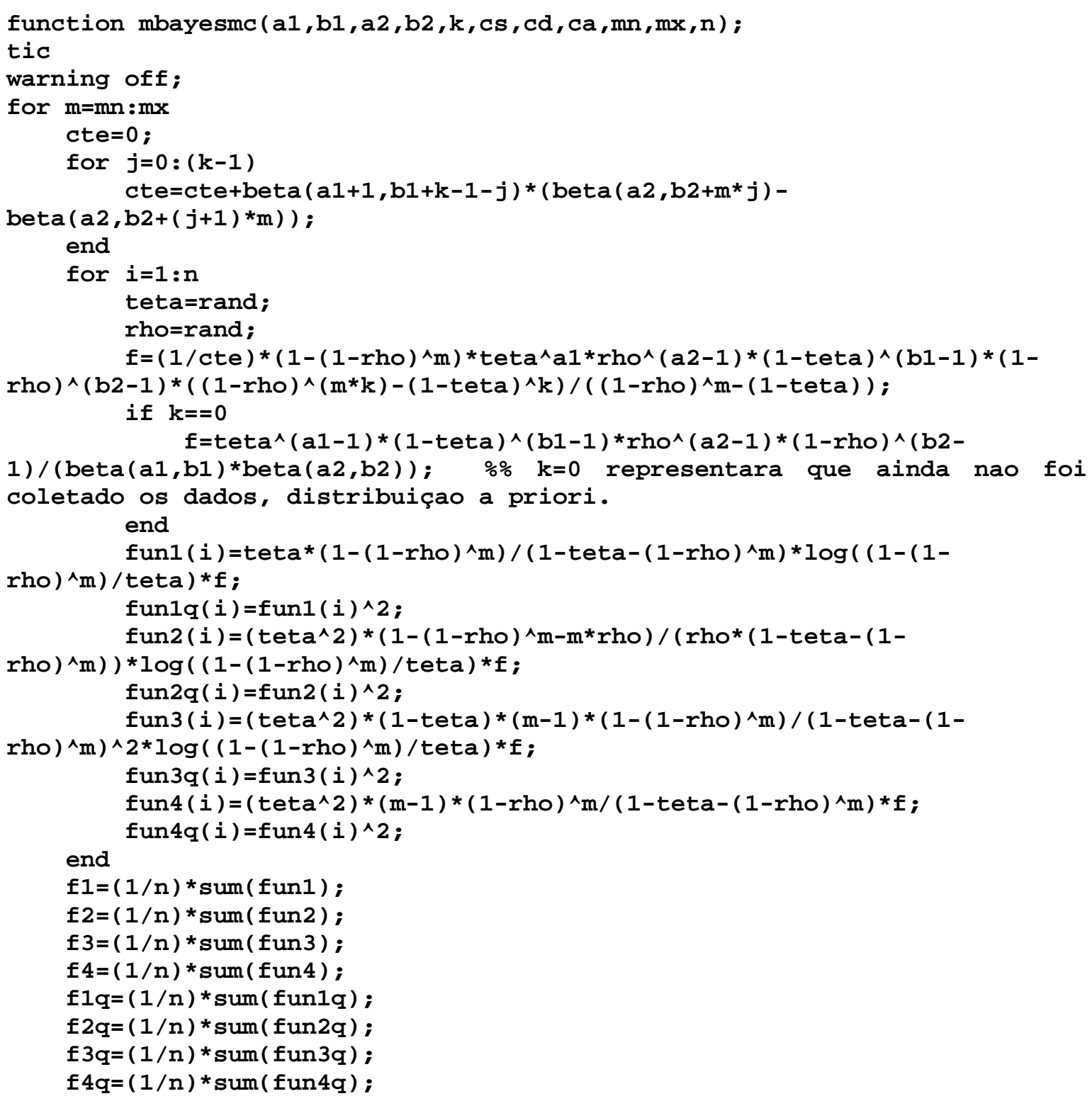


custo $(m-m n+1)=c s / m+c d^{*}(m-1) *(a 1) /\left(m^{*}(a 1+b 1)\right)+f 1 *(c a+c d) /(m)-(f 2+f 3-$ f4) ${ }^{*} \mathrm{~cd} /(\mathrm{m})$;

end

$\mathrm{m}=\mathrm{mn}: \mathrm{mx}$;

aux $=[$ custo

$\mathrm{m}]$;

aux2=sortrows (aux' ) ;

Custos=custo

M_otimo=aux2 $(1,2)$

Custo_otimo=aux2 $(1,1)$

tempo $=$ toc

plot (m, custo, ' - ')

axis([mn mx min(custo) $\max ($ custo)]) 


\section{Apêndice E}

\section{Simulação Estocástica}

Neste apêndice, apresentamos a função desenvolvida no software MATLAB R12 para implementar o algoritmo apresentado na Figura 5.4:

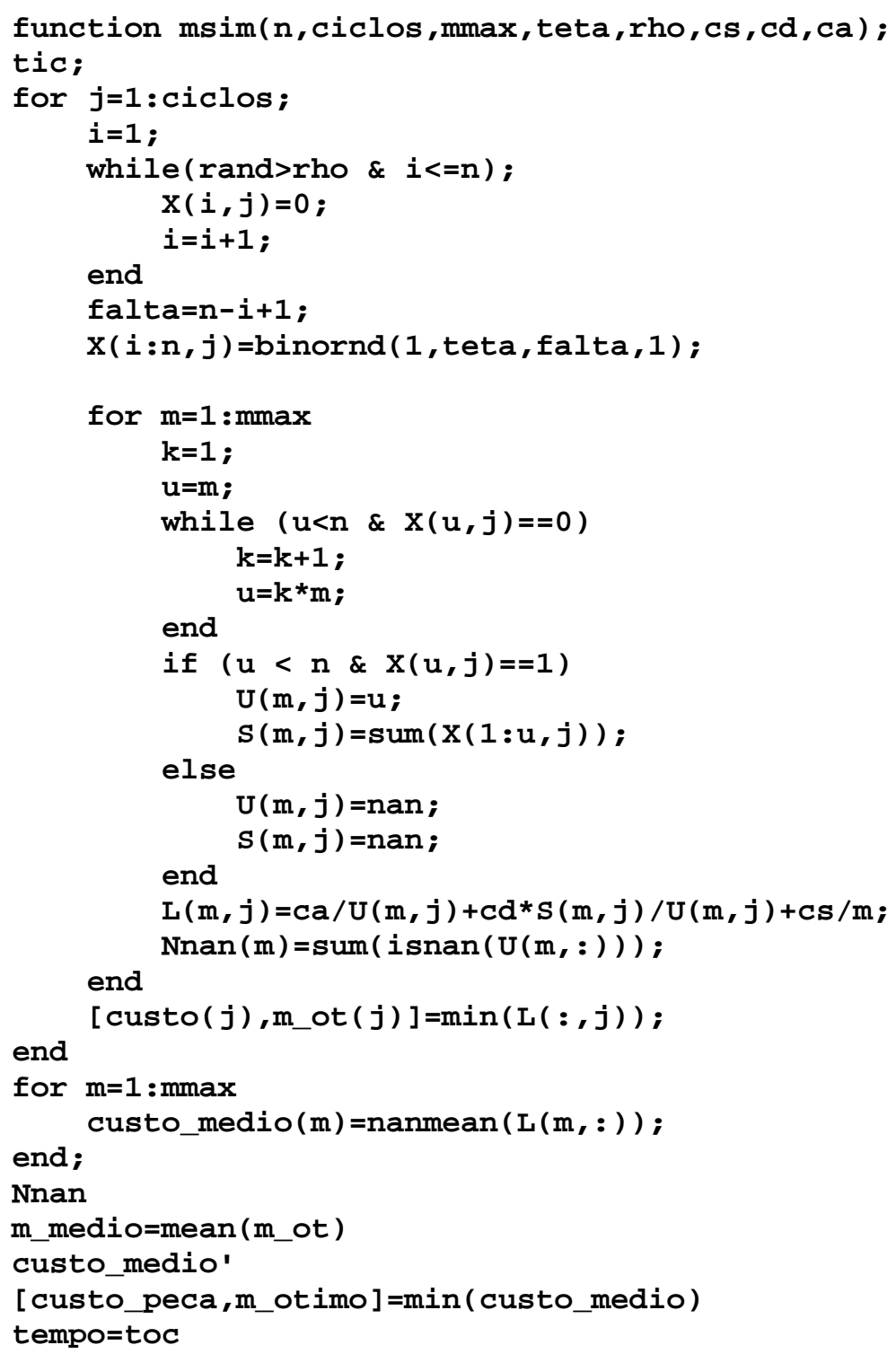




\section{Referências Bibliográficas}

[1] Belem, A. C. V. (2001). Métodos de controle de processo on-line para atributos. Dissertação de Mestrado. São Paulo: IME/USP

[2] Bernardo, J. M., Smith, A. F. M. (1994). Bayesian Theory. Chichester: John Wiley \& Sons.

[3] Borges, W. S., Esteves, L. G., Wechsler, S. (2005). Process parameters estimation in the Taguchi on-line quality monitoring procedure for attributes. São Paulo: IME-USP

[4] Casella G., Berger, R. L. (2002). Statistical Inference. 2. ed. New York: Duxbury Press.

[5] DeGroot, M. H. (1970). Optimal Statistical Decisions. New York: McGraw-Hill.

[6] Duncan, A. J. (1956). The Economic Design of X-Charts Used to Maintain Current Control of a Process. Journal of the American Statistical Association, 51, 228-242.

[7] Gamerman, D. (1997). Markov Chain Monte Carlo: Stochastic Simulation of Bayesian Inference. New York: Chapman \& Hall.

[8] Gander, W., Gautschi, W. (2000). Adaptive Quadrature - Revised. BIT, 40: 84101.

[9] Gibra, I. N. (1978). Economically Optimal Determination of the Parameters of np-Control Charts. Journal of Quality Technology, 10, 12-19.

[10] Mathews, J. H. (1992). Numerical Methods for Mathematic, Science and Engineering. 2. ed. London: Prentice-Hall International (UK).

[11] Montgomery, D. C., Heikes, R. G., Mance, J. F. (1975). Economic Design of Fraction Defective Control Charts. Management Science.

[12] Nayebpour, M. R. and Woodall, W. H. (1993). An Analysis of Taguchi's OnLine Quality-Monitoring Procedures for Attributes. Technometrics, 35: 53-60.

[13] Paulino, C. D., Turkman, M. A. A., Murteira, Bento. (2003). Estatística Bayesiana. Fundação Calouste Gulbenkian, Lisboa. 
[14] Ross, S. M. (1997). Introduction to Probability Models. 6. ed. San Diego: Academic Press.

[15] Taguchi, G. (1981). On-Line Quality Control During Production. Tokyo: Japanese Standards Association.

[16] Taguchi, G. (1984). Quality Evaluation for Quality Assurance. Dearborn, MI: American Supplier Institute.

[17] Taguchi, G. (1985). Quality Engineering in Japan. Communications in Statistics Theory and Methods, 14, 2785-2801.

[18] Taguchi, G., Elsayed, E. A., Hsiang, T. (1989). Quality Engineering in Production Systems. New York: McGraw-Hill.

[19] Williams, W. W., Looney, S. W., Peters, M. H. (1985). Use of Curtaleid Sampling Plans in the Economic Design of np-Control Charts. Technometrics, 27, 57-63. 